\title{
Testing a dynamic-field account of interactions between spatial attention and spatial working memory
}

\author{
Jeffrey S. Johnson ${ }^{1,3}$ • John P. Spencer ${ }^{2}$
}

Published online: 25 January 2016

(C) The Psychonomic Society, Inc. 2016

\begin{abstract}
Studies examining the relationship between spatial attention and spatial working memory (SWM) have shown that discrimination responses are faster for targets appearing at locations that are being maintained in SWM, and that location memory is impaired when attention is withdrawn during the delay. These observations support the proposal that sustained attention is required for successful retention in SWM: If attention is withdrawn, memory representations are likely to fail, increasing errors. In the present study, this proposal was reexamined in light of a neural-process model of SWM. On the basis of the model's functioning, we propose an alternative explanation for the observed decline in SWM performance when a secondary task is performed during retention: SWM representations drift systematically toward the location of targets appearing during the delay. To test this explanation, participants completed a color discrimination task during the delay interval of a spatial-recall task. In the critical shifting-attention condition, the color stimulus could appear either toward or away from the midline reference axis, relative to the memorized location. We hypothesized that if shifting attention during the delay leads to the failure of SWM representations, there should be an increase in the variance of recall errors, but no change in directional errors, regardless of the
\end{abstract}

Jeffrey S. Johnson

jeffrey.s.johnson@ndsu.edu

1 Department of Psychology and Center for Visual and Cognitive Neuroscience, North Dakota State University, Fargo, ND, USA

2 Department of Psychology and Delta Center, University of Iowa, Iowa City, IA, USA

3 Department of Psychology, North Dakota State University, Fargo, ND 58102, USA direction of the shift. Conversely, if shifting attention induces drift of SWM representations - as predicted by the modelsystematic changes in the patterns of spatial-recall errors should occur that would depend on the direction of the shift. The results were consistent with the latter possibility-recall errors were biased toward the locations of discrimination targets appearing during the delay.

Keywords Attention: Theoretical and computational models . Attention $\cdot$ Spatial memory

Attention and working memory are closely related cognitive processes that play an important role in everyday behaviors. For example, attention has been shown to increase the efficiency of perception, cognition, and overt behavior in complex environments by restricting cognitive processes to a taskrelevant subset of the information that is available (see the review in Luck \& Vecera, 2002). Similarly, working memory plays a role in the comparison of the perceptual information obtained from spatially and/or temporally separated objects (Hollingworth, 2009), and in maintaining information in an active state for use in complex cognitive tasks such as reading and mental rotation (Hyun \& Luck, 2007; Just \& Carpenter, 1992). Although most research has focused on examining these two processes in isolation, exploring and understanding the relationship between attention and working memory has become a central focus of research in visuospatial cognition. In particular, several lines of research have suggested a high degree of overlap between the systems of spatial working memory (SWM), on one hand, and spatial selective attention, on the other.

Decades of research on spatial selective attention has revealed that observers make faster and more accurate responses to stimuli that fall in attended versus unattended locations 
(Luck \& Vecera, 2002; Posner, 1980). Using these findings as a starting point, Awh, Jonides, and Reuter-Lorentz (1998) reasoned that if spatial attention remains focused on memorized locations during memory delays, the processing of items appearing at those locations should be facilitated relative to items appearing at different, nonmemorized locations. This is exactly what they found: Form discrimination responses were faster and more accurate when the targets appeared at locations that were being held in memory, suggesting that spatial attention remained at the memorized location during the delay interval. Critically, in an additional experiment, these researchers found that location memory was impaired when attention was shifted away from the memorized location to discriminate the color of an item appearing at a different location during the delay. This finding suggests not only that attention remains at the memorized location, but that it directly contributes to the maintenance of location information in SWM.

Functional imaging studies of SWM have likewise suggested a high degree of overlap between systems of attention and SWM (Awh et al., 1999; Postle, Awh, Jonides, Smith, \& D'Esposito, 1999). These experiments have revealed that maintaining information in SWM leads to increased activation in many of the same brain areas that are activated in experiments probing spatial attention. Additionally, event-related potential (ERP) studies of SWM (Awh, Anllo-Vento, \& Hillyard, 2000) have shown that the presentation of stimuli at locations that are being held in memory elicits ERPs with spatio-temporal patterns of amplitude enhancement similar to those observed in studies of spatial selective attention (Hillyard \& Anllo-Vento, 1998; Hillyard, Vogel, \& Luck, 1998).

On the basis of these results, Awh and colleagues (Awh \& Jonides, 1998; Awh et al., 1998) proposed the attention-based rehearsal hypothesis, which holds that, as with verbal working memory, the maintenance of information in SWM requires an active rehearsal process, and that rehearsal in SWM is mediated by spatial attention. As a result, when spatial attention is withdrawn from the memorized location to perform the color discrimination task during the delay, the representation of the target location in SWM undergoes passive decay. That is, the target location is forgotten. But in what sense is attention the rehearsal mechanism for SWM? Is continued spatial attention necessary to maintain the sustained patterns of neural activation thought to underlie working memory performance? If this were the case, one would expect severe deficits when attention is withdrawn from memorized locations to perform a secondary task. Although Awh et al. (1998) showed that performance of a same-different location memory task was impaired when attention was withdrawn to perform a secondary task during the delay, performance of the secondary task only produced a $5 \%$ drop in performance, relative to a control task that did not require a shift of attention (see Fig. 5 in Awh et al.,
1998). One might expect a more severe impairment if focused spatial attention were the mechanism responsible for maintenance in SWM.

Another possibility is that rather than being the rehearsal mechanism itself, attention instead serves to bias or modulate memory-related activity during maintenance, in keeping with its proposed role in visual perception (e.g., Desimone \& Duncan, 1995). If this were the case, shifting spatial attention might be expected to distort or bias SWM representations. Support for the idea that spatial attention biases SWM has come from several studies using variants of the spatial-recall task, in which participants are asked to estimate the location of a perceived stimulus following a short delay interval. For instance, Van der Stigchel, Merten, Meeter, and Theeuwes (2007) found that participants' memory for the location of a briefly presented target was shifted in the direction of a taskirrelevant sudden-onset distractor presented during the delay period. The distractor likely induced a transient shift in attention (similar to the shifting-control condition of Awh, et al., 1998). Similarly, other studies have revealed a compression of spatial memory toward salient central landmarks (Liverence \& Scholl, 2011) and toward the center of gaze (Sheth \& Shimojo, 2001). Additionally, Liverence and Scholl reported that attended targets in a multiple-object-tracking task are later remembered as being closer together than they actually were. These findings suggest that SWM is influenced by the focus of attention during maintenance.

In the present study, we further explored the interaction between spatial attention and SWM by combining behavioral methods with a neural process model of SWM. Specifically, we were interested in determining whether shifting attention under conditions matching those of Awh et al. (1998, Exp. 3) would induce systematic distortions of SWM representations. To do this, we conducted a behavioral experiment using their dual-task design, with two modifications: (1) We probed memory for a relatively small set of target locations (rather than randomly selecting targets from a large set) and shifted attention either toward or away from each location, relative to a midline reference axis, and (2) we used a spatial-recall task after the memory delay (rather than probing memory with a spatial discrimination task). The experiment also included a shifting-control condition, which was similar to that in the experiment conducted by Van der Stigchel et al. (2007). This design made it possible to differentiate between the effects on SWM of a reflexive shift of attention toward the location of an abrupt-onset stimulus and the voluntary allocation of spatial attention to a task-relevant target. To formalize the relationship between attention and SWM, we extended a recent model of SWM formulated within the broader theoretical framework of dynamic-field theory (DFT; Schutte \& Spencer, 2009; Spencer, Simmering, \& Schutte, 2006; Spencer, Simmering, Schutte, \& Schöner, 2007). In the following sections, we provide an overview of 
the model, followed by a series of simulations from which we derived specific predictions that would be tested in a subsequent behavioral experiment. As we will show, in addition to providing a plausible account of the reaction time (RT) advantages observed for targets appearing at memorized locations (see Awh et al., 1998, Exp. 1), the proposed model predicted a systematic drift of location memory toward the focus of attention during the delay.

\section{The DFT of spatial cognition}

The DFT is a neural-process account of visuospatial cognition that is in a class of bistable neural networks first developed by Amari and colleagues (Amari, 1977; Amari \& Arbib, 1977; see also Camperi \& Wang, 1998; Compte, Brunel, GoldmanRakic, \& Wang, 2000; Edin et al., 2009). This approach provides a basis for characterizing the representational states underlying behavior through the concept of activation, which is familiar from mathematical psychology, connectionism, and theoretical neuroscience (Churchland \& Sejnowski, 1992; Williams, 1986). In the dynamic-field approach, this concept takes the form of an activation field, defined over the metric dimension represented. For example, in a typical location working memory task, activation would be distributed across a continuous, metric spatial dimension (e.g., target direction) encompassing the area on the computer monitor where memory targets could appear. A localized peak of activation within this field would indicate that a target object (e.g., a small black circle) had been detected at a particular location in the task space. Patterns of activation within such fields can live in different attractor states, including a stable resting state, in which activation remains at baseline levels for all field sites; an input-driven state, in which input can form stabilized "peaks" of activation that represent the estimates of particular stimulus parameters (e.g., location, color), but that relax back to the resting state when input is removed; and a self-sustaining state, in which the activation peaks remain stable in the absence of continued input - a form of working memory central to our work, and the main focus of the present article.

Figure 1 shows a simulation that implements the core features of the DFT of spatial cognition (for discussion of the full model, see Spencer et al., 2007). The model consists of three layers: a perceptual, or input, layer (PF; Fig. 1A), a layer of inhibitory interneurons (Inhib; Fig. 1B), and a spatial working memory layer (SWM; Fig. 1C). In each layer, the direction of targets in the task space is shown along the $x$-axis (left-right); the $y$-axis (up-down) shows the activation of each neuron in the field; and the elapsed time from the start to the end of the trial is shown along the $z$-axis, with time moving from front to back. Arrows depict excitatory (solid) and inhibitory (dashed) connections among the fields.
One of the most common tasks used to examine SWM is spatial recall. In this task, participants are briefly shown a single target item and are asked to remember the target's location. This is followed by a delay interval of variable length $(0-20 \mathrm{~s})$, after which the participant indicates the remembered location of the target by, for instance, moving a computer mouse or a joystick. A number of studies have demonstrated that metric memory for location shows delay-dependent biases away from the perceived reference axes, such as the midline symmetry axis of a task space (see, e.g., Engebretson \& Huttenlocher, 1996; Huttenlocher, Hedges, \& Duncan, 1991; Schiano \& Tversky, 1992; Schutte, Spencer, \& Schöner, 2003; Spencer \& Hund, 2002; Tversky \& Schiano, 1989; Werner \& Schmidt, 2000). Before proceeding to a description of the model simulations for the present study, it will be helpful to walk through the simulation shown in Fig. 1, which illustrates how the spatial-recall task is implemented in the model and how biases away from the perceived reference axes are explained. Walking through this simulation will set the stage for a discussion of how attention may serve to bias the patterns of activation in SWM.

At the start of the trial, PF builds a small peak of activation at $0^{\circ}$, reflecting the perception of a salient reference frame in the environment (e.g., two dots along the vertical midline of the task space; see the stimulus displays in Fig. 2A). Next, a target appears at $-40^{\circ}$. This creates a peak of activation centered at that location. When the target disappears, a peak re-forms in PF at $0^{\circ}$ as the system relocks onto the reference cues in the task space. This is important, because it allows the system to continually calibrate the egocentric and object- or table-centered frames of reference during the delay (Spencer et al., 2007).

Panel C shows the effect of coupling PF to SWM. At the start of the trial, SWM receives relatively weak reference input from $\mathrm{PF}$ because the reference cue in the task space (i.e., the reference input to PF) is not very salient. Next, the target is turned on, passing strong target-related input into SWM. This event moves the working memory layer into a self-sustaining state in which peaks of activation can be maintained in the absence of input.

Maintenance is made possible in the model through reciprocal coupling between the SWM and Inhib layers, which implements a locally excitatory and laterally inhibitory form of interaction among neurons (Compte et al., 2000). Specifically, the neurons in SWM have locally excitatory interactions that boost activation of the neurons tuned to similar spatial locations. Additionally, SWM is reciprocally coupled to the layer of inhibitory neurons: The neurons in SWM provide excitatory inputs to similarly tuned neurons in Inhib, which, in turn, project inhibition broadly back to SWM. This pattern of interactions allows localized, self-sustained peaks of activation to be maintained in SWM in the absence of continued input (e.g., when the target object is removed from view).

Importantly, the activation peak in SWM in Fig. 1 is stably maintained during the memory delay, even though PF has reacquired the reference frame. This sets the stage for 


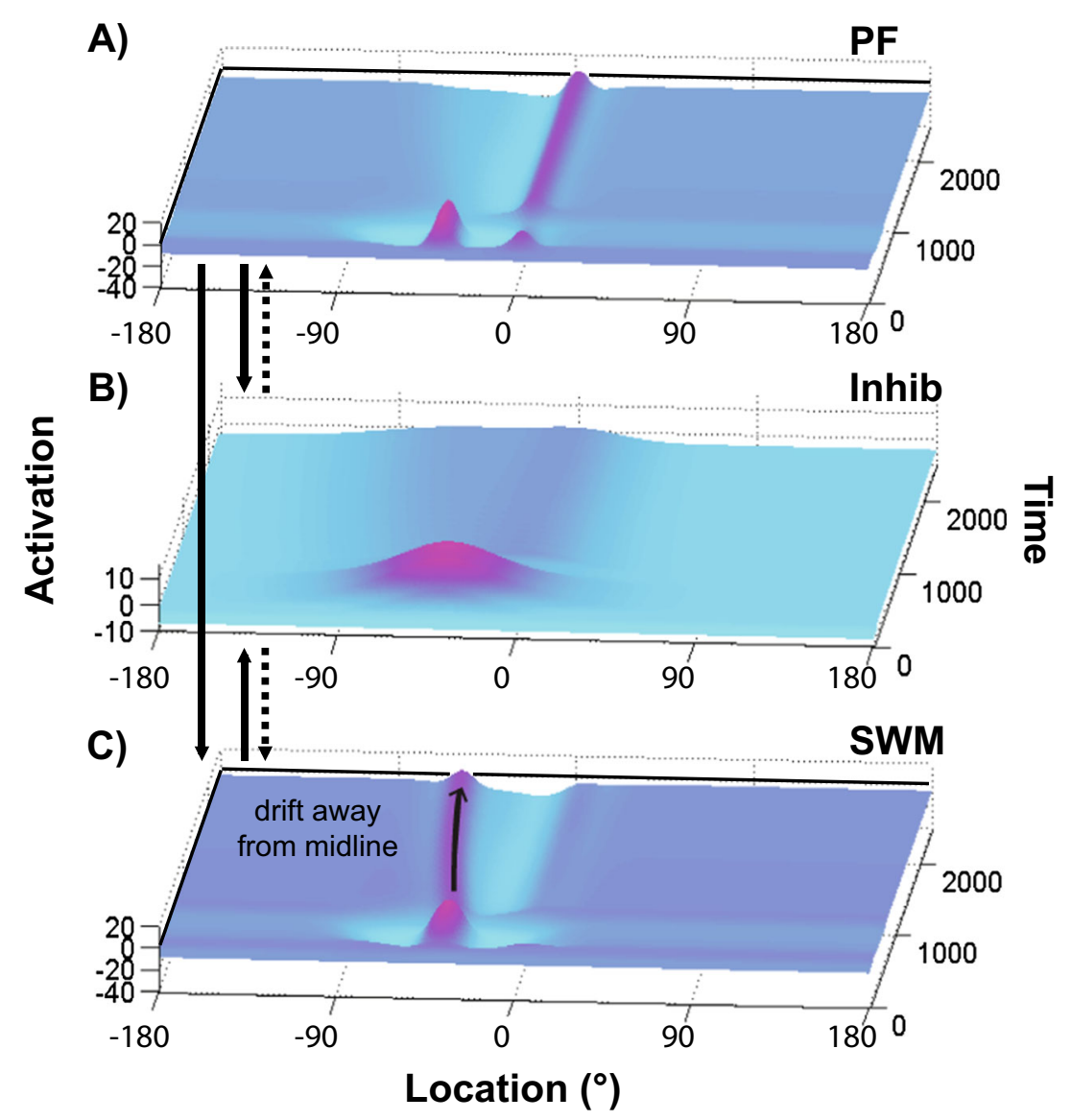

Fig. 1 Simulation of the dynamic-field theory (DFT) performing a single trial in a spatial-recall task. Weak, reference-related input to the PF layer of the model, reflecting perception of the midline symmetry axis of the

memories to drift away from the reference frame because the system is effectively holding onto two stable states at once: It is locked onto the reference frame in PF to stay calibrated with the world, and it is actively maintaining a memory of the target location in SWM. The response bias that is evident in experiment arises from the place where these two stable states meet - the shared inhibitory layer. In particular, referencerelated input to the inhibitory layer near midline produces a region of inhibition at this same location in SWM. This causes the peak of activation in SWM to "drift" away from the vertical midline of the task space, because inhibition is stronger on the midline side of the SWM peak than on the "outer" side. In effect, the reference frame "pushes" the peak outward, exaggerating the "leftness" of the target location.

\section{Testing the DFT: Do shifts in attention cause SWM to spatially drift?}

The simulation described in the previous section illustrates two phenomena that have been shown to coexist in spatial recall: the sustained activation of location-specific neural populations throughout the memory delay, and the systematic drift of these task space, produces delay-dependent drift of spatial working memory (SWM) representations away from the midline

neural patterns away from a perceived reference axis. The latter effect arises from the tendency for activation peaks to drift along activation gradients, moving from regions of relatively strong inhibition to areas of weaker inhibition - that is, toward the field locations where excitation is strongest (Amari, 1977; Spencer, Perone, \& Johnson, 2009; Wang, 2001). In most cases, this property ensures that the peak of activation is centered over the region of maximal stimulation, corresponding to the likely position of the stimulus input (a formal analysis of this property of neural fields can be found in Amari, 1977). In other situations, however, this can lead to systematic distortion, as with repulsion from reference frames. As another example, if participants are asked to repeatedly estimate a small set of locations, spatial-recall responses are biased toward the center of the distribution of targets presented throughout the experimental session (see, e.g., Hund \& Spencer, 2003; Lipinski, Simmering, Johnson, \& Spencer, 2010; Spencer \& Hund, 2002). These findings have also been modeled with DFT using a memory trace mechanism. In this case, memory traces build up across trials, driven by activation peaks in working memory. These memory traces feed back into working memory as weak excitatory inputs, biasing working memory toward previously 


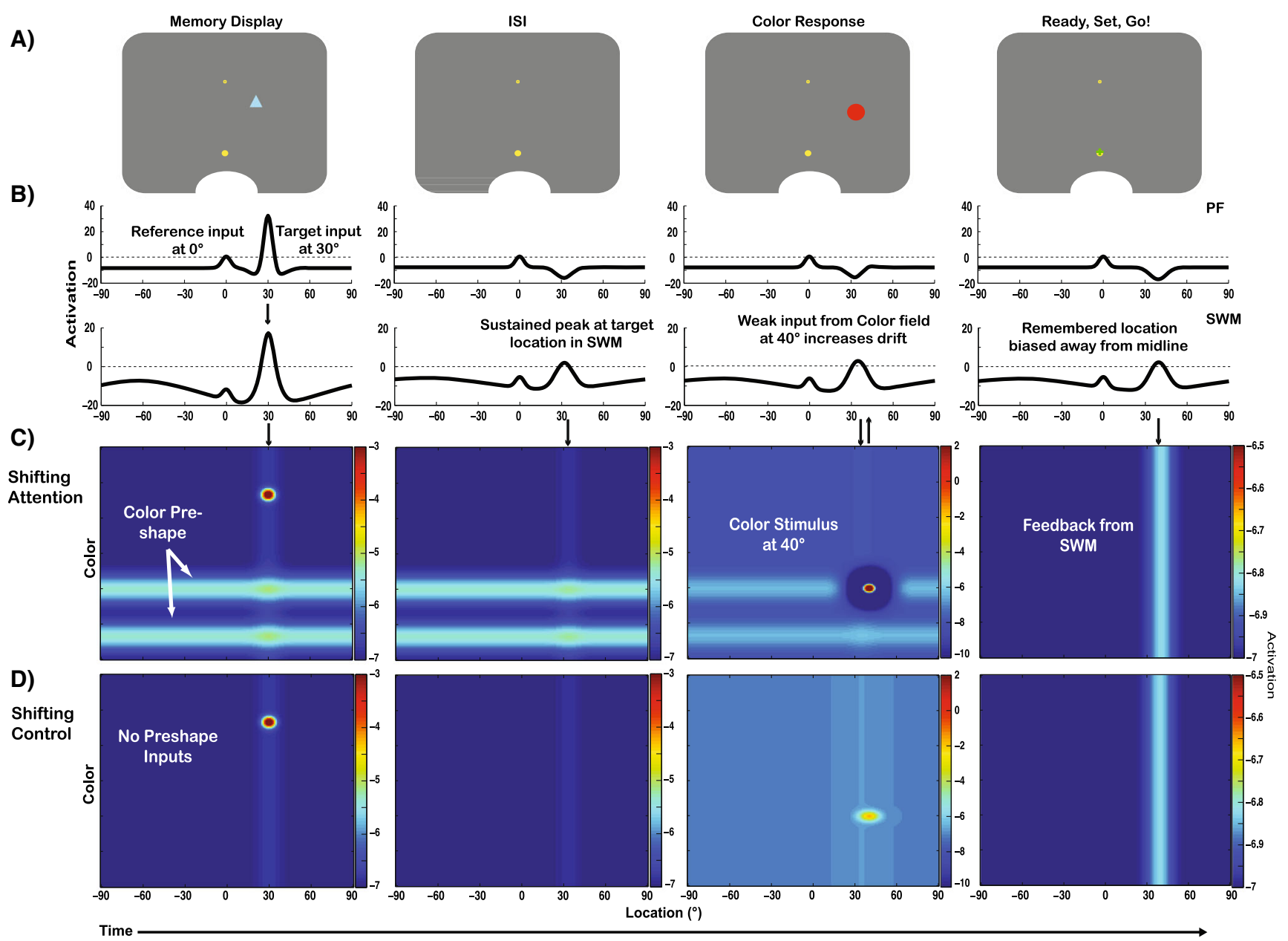

Fig. 2 Simulation of the model performing a single trial in the shiftingattention and shifting-control conditions. Panel $\mathbf{A}$ depicts the events in the simulated experiment; the memory target is presented at $30^{\circ}$ in the task space, followed by a color discrimination target presented $10^{\circ}$ farther away from midline (i.e., at $40^{\circ}$ in the task space). Panel $\mathbf{B}$ shows the patterns of activation in the PF (top) and SWM (bottom) layers of the model at time points corresponding to the task events depicted in panel $\mathbf{A}$.

remembered locations (Lipinski, Spencer, \& Samuelson, 2010; Spencer et al., 2007). ${ }^{1}$

In the present study, we examined whether a similar mechanism could explain an additional effect - the drift of SWM representations toward the focus of attention. This would be the case if, for example, shifting spatial attention served as an additional source of location-specific excitatory input to SWM, modulating the patterns of sustained activation during a trial in a manner

\footnotetext{
${ }^{1}$ Note that the memory trace mechanism described here was not included in the present set of simulations. To control for the possibility of longterm-memory (LTM)-based effects on recall, we adopted the strategy of presenting a small number of locations at fixed positions relative to midline, and participants were exposed to each location an identical number of times throughout the experiment. As a result, the LTM traces formed for the different locations would have been identical, and thus, any observed effects of discrimination-target direction on the recall estimates could not have been due to LTM effects of the sort described here.
}

Panels $\mathbf{C}$ and $\mathbf{D}$ show the patterns of activation in the CS field for the shifting-attention and -control conditions, respectively. The preshape input to the CS field in the shifting-attention condition (C) leads to the formation of a strong peak of activation during color discrimination. As a result, excitatory input from the color-space field to SWM at the location of the color target increases drift of the working memory peak away from the midline reference input. See the text for additional details

similar to the memory trace effects discussed above. To implement this idea, we extended the three-layer model of SWM to capture performance in a shifting-attention condition, in which a color discrimination task requiring a shift of spatial attention was performed during the delay, and a shifting-control condition, in which the discrimination stimulus appeared, but no response had to be made (as in Van der Stigchel et al., 2007). In keeping with previous results in this area, the results of model simulations suggested that spatial selective attention can indeed distort working memory for locations. In a subsequent behavioral experiment, we then tested specific predictions of the dynamic-field account.

\section{Model simulations}

In this section, we adapt the DFT of spatial cognition to examine how shifts of spatial selective attention during memory 
delays affect SWM. In particular, we coupled the three-layer model of SWM to a single, 2-D color-space (CS) field that represented both the color and the spatial location of targets (see Fig. 2). The model captures the assumption that performance of the color discrimination task requires the short-term maintenance of color information, and that this occurs via sustained activation within a spatially organized color system. The model is structured such that the spatial dimension of the color system sends location-specific excitatory input to SWM, which allows information regarding the location of an attended color target to influence the activation in SWM. Although representing the location of the color discrimination stimulus was not strictly required by the task - that is, participants were never asked to localize the discrimination targetorganizing the model architecture in this way was reasonable, given that many ventral visual cortical areas with cells that respond selectively to color are also spatially organized (see, e.g., Bartels \& Zeki, 2000; Xiao, 2014; Xiao, Wang, \& Felleman, 2003). Moreover, many of these same areas have been found to send projections to dorsal-stream areas that have been implicated in working memory for spatial information (Claeys et al., 2004). The simulation results reveal that weak spatial input from the CS field to SWM during discrimination induces a bias toward the focus of attention during the delay.

\section{Method}

Model architecture The model used to simulate the shiftingattention and shifting-control conditions built on the threelayer dynamic-field model of SWM described above. As noted, we added a single CS field to this architecture. This field implemented a simple form of decision making, forming a sustained peak of activation in response to task-relevant color inputs during the delay. The CS field was bidirectionally coupled to the SWM layer of the three-layer model.

Each layer of the three-layer model contained 181 bistable neural sites with receptive fields tuned to particular locations in the task space. The spatial location of targets in the task space was specified in degrees of angular rotation away from the vertical midline $\left(0^{\circ}\right)$, with counterclockwise targets being given a negative sign, and clockwise targets, a positive sign (see Fig. 1). Interactions between the SWM and Inhib layers made it possible for sustained peaks of activation to be maintained in SWM in the absence of continuing input (i.e., once the stimulus input had been turned off). The perceptual inputs to the model took the form of a Gaussian centered at a particular location in the PF field. Projections among the different layers (e.g., from PF to Inhib and/or SWM) were defined by the convolution of a Gaussian kernel with a sigmoidal threshold function. The threshold function determined the degree to which neural sites close to threshold (i.e., 0 ) contributed to the activation dynamics (see the Appendix and Table 1 for the model equations and parameters).

The CS field consisted of a $181 \times 181$ topographic array of neural sites. Sites in this field responded preferentially to specific colors appearing at particular locations in the task space. For example, whereas one region of this field might respond to red targets appearing at $40^{\circ}$ of angular rotation from the vertical midline in the task space, another nearby region might respond to red targets appearing at $45^{\circ}$, and so forth. This architecture represented a simplification of the known properties of color-selective regions of the visual cortex, in which a given neuron's firing rate is determined by its coordinates in three dimensions: two dimensions of visual space and one dimension of hue. Although we made use of threedimensional dynamic fields in both the behavioral models and robotic architectures (Schneegans, 2016; Zibner \& Faubel, 2016), here we consider only a single spatial dimension. In this case, activation along the spatial dimension can be thought of as reflecting the angular position relative to the vertical midline of a stimulus appearing at a fixed distance from fixation. In addition to simplifying the representation of space, the CS field was implemented as a single-layer neural field. In this simplified color system, activation evolved over time according to a single field equation in which excitatory interactions dominate for nearby field locations, and inhibitory interactions dominate at more distant field sites. Additionally, input was added directly into the CS field, rather than being transmitted from an input layer, as in the threelayer SWM model. This simplified architecture retained the functional properties of more complex candidate models, while reducing the computational load and facilitating the illustration and description of activation patterns.

Inputs to the CS field took the form of a Gaussian in two dimensions (color and space). Although the width of the Gaussian could be set to different values for each dimension (e.g., narrow in feature and broad in space), for simplicity, here we use the same value along each dimension. To implement decision making in the CS field, neurons responding to the colors used in the color discrimination task were provided with weak subthreshold "preshape" inputs, a form of featurebased attention. Preshape inputs take the form of a ridge of activation that is localized along the color dimension but not the spatial dimension, providing low levels of spatially global excitatory input to the neural sites coding for particular colors (Liu \& Mance, 2011; Maunsell \& Treue, 2006; Serences \& Boynton, 2007). Such inputs are much weaker than direct stimulus inputs and cannot by themselves induce a localized peak of activation. Additionally, the CS field was tuned so that above-threshold peaks of activation were only possible when a color matching one of the preshaped values (i.e., one of the possible color targets) was presented to the model. This prevented the formation of above-threshold peaks in the CS field in response to the memory target (which was a different 
Table 1 Parameter values for simulations

\begin{tabular}{|c|c|c|c|c|c|c|c|c|}
\hline Layer & $\tau$ & $h$ & Self-Excitation & Excitatory Projection(s) & Inhibitory Projection(s) & Reference Input & Preshape Input & Target Input \\
\hline$u(\mathrm{PF})$ & 80 & -7 & $\begin{array}{l}c_{\mathrm{uu}}=1.75 \\
\sigma_{\mathrm{uu}}=5\end{array}$ & & $\begin{array}{l}c_{\mathrm{uv}}=1.1 \\
\sigma_{\mathrm{uv}}=5\end{array}$ & \multicolumn{2}{|l|}{$\begin{array}{l}c_{\text {ref }}=8 \\
\sigma_{\text {ref }}=3\end{array}$} & $\begin{array}{l}c_{\mathrm{tar}}^{\mathrm{a}}=40 \\
\sigma_{\mathrm{tar}}=3\end{array}$ \\
\hline$v$ (Inhib) & 10 & -12 & & $\begin{array}{l}c_{\mathrm{vu}}=4.88 \\
\sigma_{\mathrm{vu}}=5 \\
c_{\mathrm{vw}}=2.2 \\
\sigma_{\mathrm{vw}}=6\end{array}$ & & & & \\
\hline$w(\mathrm{SWM})$ & 80 & -4 & $\begin{array}{l}c_{\mathrm{ww}}=2.0 \\
\sigma_{\mathrm{ww}}=6\end{array}$ & $\begin{array}{l}c_{\mathrm{wu}}=1.5 \\
\sigma_{\mathrm{wu}}=3 \\
c_{\mathrm{wwc}, \mathrm{s}}=0.04 \\
\sigma_{\mathrm{wwc}, \mathrm{s}}=6\end{array}$ & $\begin{array}{l}c_{\mathrm{wv}}=0.615 \\
\sigma_{\mathrm{wv}}=40 \\
k_{\mathrm{wv}}=0.05\end{array}$ & \multicolumn{2}{|c|}{ [all inputs scaled by $c_{\mathrm{s}}=0.2$ ] } & \\
\hline$w_{c, s}(\mathrm{CS})$ & 80 & -7 & $\begin{array}{l}c_{\mathrm{wc}, \mathrm{swc}, \mathrm{s}}=1.69 \\
\sigma_{\mathrm{wc}, \mathrm{swc}, \mathrm{s}}=3\end{array}$ & $\begin{array}{l}c_{\mathrm{wc}, \mathrm{sw}}=0.025 \\
\sigma_{\mathrm{wc}, \mathrm{sw}}=6\end{array}$ & $\begin{array}{l}c_{\text {inhib,xy }}=1.25 \\
\sigma_{\text {inhib,xy }}=9 \\
w_{\text {inhib,xy }}=0.10\end{array}$ & & $\begin{array}{l}c_{\text {pre }}{ }^{\mathrm{b}}=12 \\
\sigma_{\text {pre }}=8\end{array}$ & $\begin{array}{l}c_{\mathrm{tar}}=5.5 \\
\sigma_{\mathrm{tar}}=5\end{array}$ \\
\hline
\end{tabular}

\footnotetext{
${ }^{\mathrm{a}}$ These values reflect target input strength to $u$ for the spatial-recall task. For the color discrimination task, input to $u$ was scaled by $0.125 .{ }^{\mathrm{b}}$ Note that preshape input was only provided to the CS field in the shifting- and static-attention conditions, in which participants were required to complete the color discrimination task during the delay interval.
}

color than the colors used in the color discrimination task), and to color targets in the control conditions, in which the color stimulus was to be ignored.

The bidirectional coupling between CS and SWM was defined by the convolution of a Gaussian kernel with a sigmoidal threshold function. Thus, whenever an above-threshold peak was present in CS, weak excitatory input was transmitted to neural sites tuned to the same location in the SWM field. Similarly, an above-threshold peak in SWM provided weak excitation to the neural sites coding the same location in the CS field. This input was propagated across all neural sites tuned to the stimulated location, regardless of their colorspecific tuning. Thus, this input produced a "ridge" of activation centered at the memorized location in the CS field, effectively priming the CS field to respond to targets appearing at those locations, regardless of their color.

Simulation details and example simulations The simulations were conducted in MATLAB 7.4 (The MathWorks, Inc.) on a PC with an AMD Athlon 2.61-GHz dual-core processor (the MATLAB code is available from the authors on request). The dynamic-field equations (see the Appendix and Table 1) were integrated using the Euler method with one time step ( $=2 \mathrm{~ms}$ ). This time step was sufficiently small that the numerical solutions approximated real solutions of the dynamical system, given a target presentation of $2 \mathrm{~s}$ and a delay interval of $10 \mathrm{~s}$. For the present simulations, our goal was to determine the impact of excitatory input to SWM on recall responses in conditions corresponding to the shiftingattention and shifting-control conditions of Awh et al. (see Fig. 4 in the Experiment section of the present study). To do this, we simulated four separate trials of each condition (shifting vs. control), with memory targets presented either $30^{\circ}$ or $60^{\circ}$ away from the vertical midline (see the $30^{\circ}$ target trial shown in Fig. 2A) and the discrimination target presented either $10^{\circ}$ closer to (shift-toward condition) or $10^{\circ}$ farther away from (shift-away condition) midline than the target (i.e., at either $20^{\circ}$ or $40^{\circ}$ for the $30^{\circ}$ target conditions, and either $50^{\circ}$ or $70^{\circ}$ for the $60^{\circ}$ target condition).

Example simulations are shown in Fig. 2, which depicts the patterns of activation in the PF, SWM, and CS fields during four different epochs of the shifting-attention and -control conditions (shift-away trial shown): memory encoding, maintenance, color discrimination, and recall. Panel A shows the displays "seen" by the model at each point in the simulated trial. Panel B shows the PF and SWM layers of the three-layer model, with spatial location along the $x$-axis and activation level on the $y$-axis (for simplicity, the Inhib layer is not shown). Finally, panels C and D show the 2-D patterns of activation in the CS field at each time point in simulations of the shifting-attention and shifting-control conditions, respectively, with activation denoted by color, with warmer colors representing greater activation (note that the color scale was adjusted for each plot to accentuate the patterns of activation at each time point). Each simulation began with a 200 -ms relaxation period to allow the model to reach a stable resting state. This was followed by the 2-s presentation of a memory display, accompanied by an additional, weaker reference input to PF that captured perception of the vertical midline of the task space (the midline input to PF stayed on throughout the remainder of the simulated trial). In addition, for the shiftingattention condition, neural sites in the CS field representing the two possible target colors in the color discrimination task received weak preshape inputs that moved sites tuned to these colors closer to threshold (see the horizontal lines in the leftmost panel of Fig. 2C). By contrast, in the shifting-control condition, no preshape inputs were provided (cf. the leftmost 
panels of Fig. $2 \mathrm{C}$ and D). Aside from this difference, these conditions were identical.

Presentation of the memory display was followed by a 5-s unfilled interstimulus interval (ISI), during which a selfsustained peak of activation, reflecting the spatial location of the memory stimulus, was maintained in SWM (Fig. 2B, SWM layer, second column). Next, one of two possible color stimuli was presented $40^{\circ}$ from midline (i.e., $10^{\circ}$ away from midline, relative to the target) for $500 \mathrm{~ms}$ (Fig. 2, third column). In both conditions, this event produced an abovethreshold peak of activation in the CS field at coordinates corresponding to the spatial position and color of the discrimination target, and relatively weak location-specific excitation in PF. Note that, due to the absence of preshape input, the resulting peak was much weaker in the shifting-control than in the shifting-attention condition (cf. Fig. 2C and D, third column). As a result, for the shifting-control condition, activity in the CS field quickly relaxed back to the resting level once the color input was turned off. In the shifting-attention condition, however, a peak of activation remained in CS until the preshape input was turned off $500 \mathrm{~ms}$ later. Prolonged preshape input to the CS field ensured that color-related activity would be present in CS long enough for a color discrimination response to be made. Finally, by the end of the 5-s ISI following color-stimulus offset (not shown), the only activation present in the CS field reflected excitatory feedback from SWM (Fig. 2C and D, rightmost panels). Spatial-recall errors were determined by computing the difference between the location of maximum activation in the SWM field at the end of the delay and the location of the original memory stimulus.

\section{Results}

The simulation results are shown in Fig. 3A. Recall that for the control conditions, the three-layer model received weak, localized input reflecting the position of the color discrimination stimulus, but no additional input from the CS field (because no above-threshold peak was present in the CS field following color-stimulus onset). This produced a small bias in recall in the direction of the color stimulus (i.e., away vs. toward midline).

In the shifting-attention conditions, the presence of color preshape input allowed a peak of activation to form in CS in response to the color stimulus. Because of this, localized excitatory input was transmitted from the CS field to SWM during the discrimination of the target color. This resulted in a robust effect of the color stimulus on SWM: When the color stimulus was presented away from midline relative to the remembered location, drift increased by $3.0^{\circ}$ relative to when the color stimulus was presented toward midline (cf. the black solid and dashed lines in Fig. 3A). Similar attention-related modulations of drift were observed for both the $30^{\circ}$ and $60^{\circ}$ targets, although the overall level of drift away from midline was reduced in the $60^{\circ}$ conditions, in keeping with the findings of behavioral studies of spatial recall (see, e.g., Spencer \& Hund, 2002).

Direct spatial interactions between SWM and CS also made it possible to capture a second key effect reported by Awh et al. (1998): faster RTs to targets appearing at memorized versus nonmemorized locations. An example simulation illustrating how this finding might be captured by the DFT is shown in Fig. 4. Figure 4A shows the SWM layer of the threelayer model during maintenance of a memory target at $30^{\circ}$ in the task space. The lower panels show the patterns of activation in the CS field on a single trial when a discrimination target was presented at either a memorized location (Fig. 4B) or a nonmemorized location (Fig. 4C). The horizontal bands of activation in each CS field reflect preshape activation at the two possible discrimination target color values, as in the simulations described above. Finally, to more clearly highlight the effect of target location (memorized, nonmemorized) on the buildup of activation in CS, Fig. 4D shows the rise time of activation at specific positions in the CS fields representing the color and spatial location of the discrimination target presented on that trial. The time it takes for activation to reach a discrimination threshold under different conditions can be used as a proxy for RT in the model. As can be seen in Fig. 4D, activation in the CS field reached threshold (i.e., 0) faster when the discrimination target appeared at the memorized rather than at the nonmemorized location, in keeping with the behavioral observations of Awh et al. (1998).

\section{Discussion}

An extension of the dynamic-field model of SWM was used to implement a recall variant of the shifting-attention and control conditions of Awh et al. (1998). Simulation results revealed that SWM was biased in the direction of a color target that had to be discriminated during the delay. This effect was observable as a modulation of the magnitude of drift away from midline as a function of the direction of attention: The spatial drift was greater when attention was shifted away from midline, and reduced when attention was shifted toward midline. This effect arises as a result of weak excitatory input that is transmitted from the spatial dimension of the CS field to SWM at the location of the discrimination target. This produces a region of relatively greater excitation at field sites in SWM corresponding to the location of the discrimination target. As a result, the peak in SWM, which represents the spatial location of the recall target, moves along the excitatory gradient in the direction of the discrimination target. When the discrimination target appears away from midline relative to the target, the drift away from midline is amplified because the peak is simultaneously pushed away from midline by reference-related inhibition and attracted toward the 

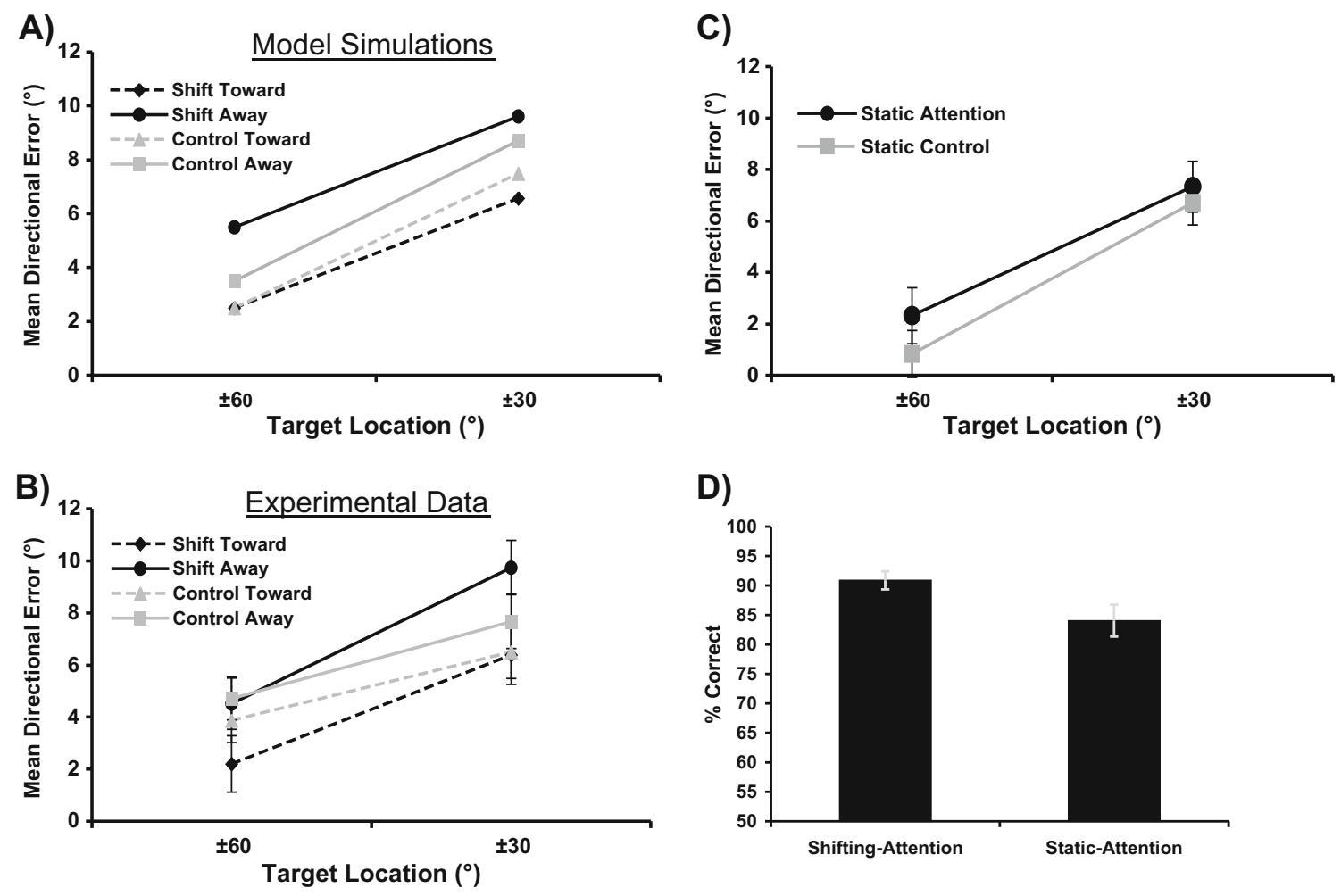

D)

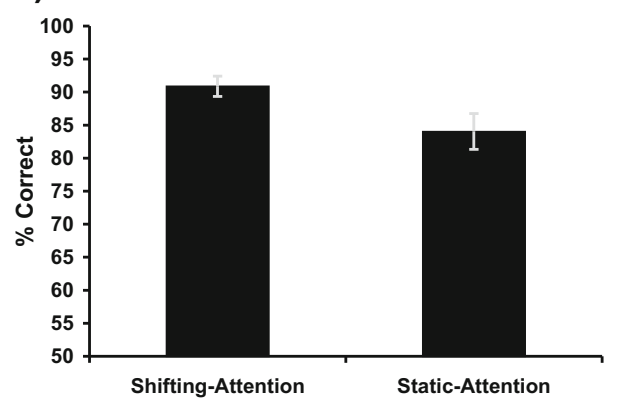

Fig. 3 Experimental data and results from the model simulations. (A) Model simulation results. (B-C) Behavioral results showing performance in (B) the shifting-attention and shifting-control conditions and $(\mathbf{C})$ the

static-attention and static-control conditions. (D) Discrimination performance in the shifting- and static-attention tasks

excitatory spatial input from CS. When the discrimination target appears toward midline, the drift away from midline is reduced because the excitatory input on the midline side of the peak counteracts the inhibition associated with the midline reference input, weakening reference-related drift. A qualitatively similar, though smaller, effect was also observed in the shifting-control condition. This effect was much smaller, due to the fact that, in the absence of preshape input, no peak formed in CS in response to the (task-irrelevant) color stimulus. This greatly reduced the strength of the activation transmitted from CS to SWM during the delay. Thus, the effect observed here depended on the spatial interactions between CS and SWM, which were enabled by a mechanism akin to feature-based attention (i.e., preshape input). Note that this same form of coupling made it possible to account for the finding of faster RTs for targets appearing at memorized than at nonmemorized locations.

Spatially specific input to SWM represents a candidate mechanism that may help explain the attention-related effects on SWM found in other contexts (Liverence \& Scholl, 2011; Sheth \& Shimojo, 2001; Van der Stigchel et al., 2007). Critically, our simulations suggest an alternative to the attentionbased rehearsal hypothesis proposed to account for the decline in SWM performance observed in previous studies. In the following section, we tested the predictions of the dynamic-field account.

\section{Experiment}

To determine whether the modulations of drift observed in the simulation experiment would also occur in human observers, we conducted an experiment in which participants completed the location memory task depicted in Fig. 5. In this task, participants were shown a single target item and had to remember the target location across a subsequent 10-s delay interval. In addition to the shifting-attention and shifting-control conditions included in the model simulations, we also included static-attention and static-control conditions, in which the color stimuli were large colored circles that covered a large portion of the task space, including all possible target locations (see Fig. 5). In these conditions, color information was available throughout the task space, and no shift of attention was required for accurate discrimination performance. As in Awh et al. (1998), this condition was included to ensure that any observed effects of the color discrimination task were in fact due to shifting attention. Our model made no specific predictions regarding performance in the static conditions.

This design allowed us to test two competing ideas about how shifting attention impacts memory for individual locations. The first idea was consistent with the proposal of Awh and colleagues (1998), and suggested that shifting attention leads to a global reduction in the stability of working memory maintenance. According to this view, attention is 


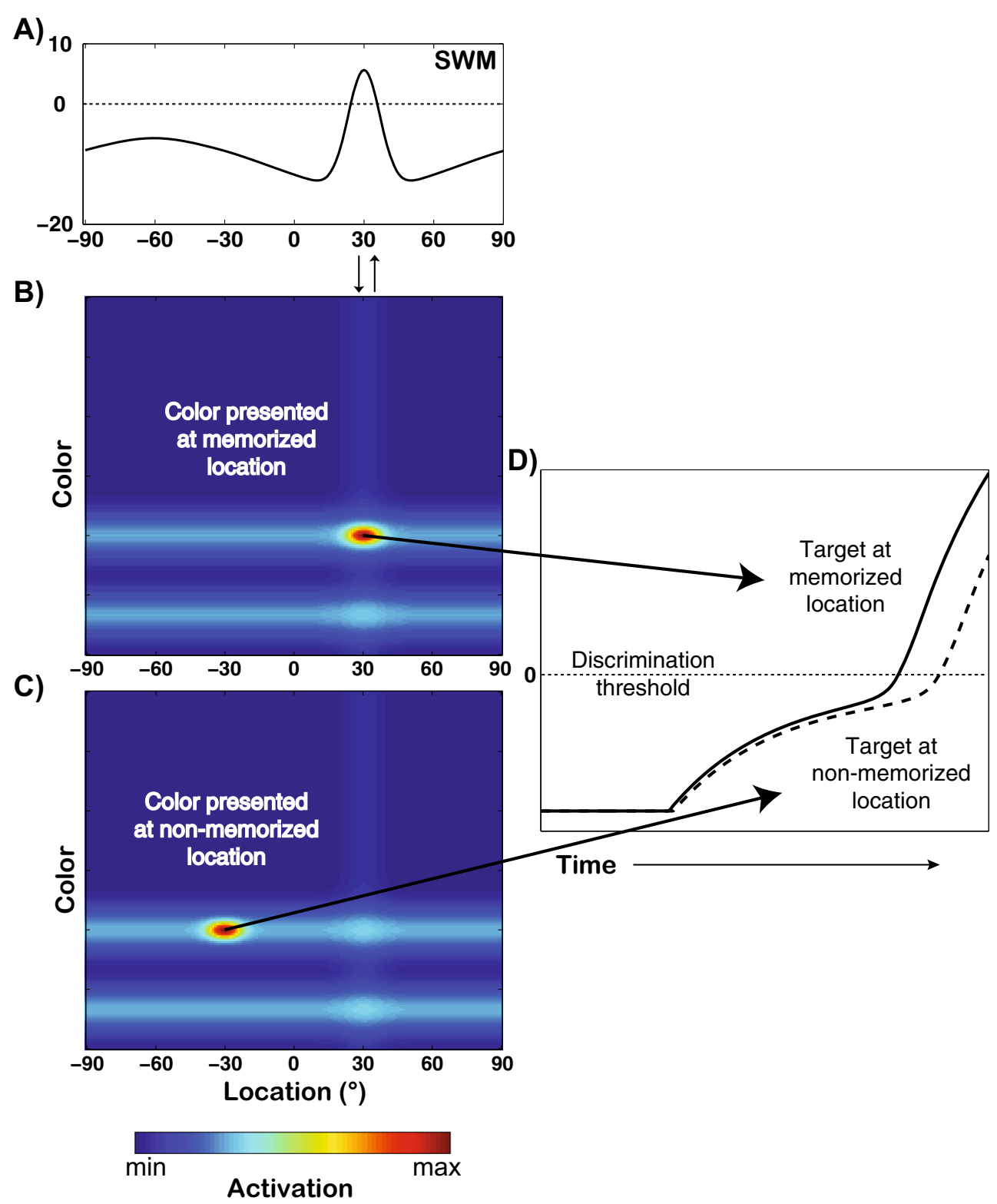

Fig. 4 Simulation showing faster rise times for activation at memorized than at nonmemorized locations in response to the color target in a color discrimination task. The storage of a peak in SWM (A) produces a ridge of activation along the spatial dimension of the CS field (B-C). This

allows activation in the CS field to build more quickly when a discrimination target is presented at this location rather than a nonmemorized location (D). See the text for additional details fundamentally tied to the rehearsal mechanism in SWM. Therefore, if attention is directed elsewhere, location memory should suffer. In particular, if shifting attention destabilizes SWM representations, we would expect to observe an increase in the variance of recall responses, but no systematic shift in the directionality of the errors across shifting-attention conditions (i.e., whether attention was shifted toward or away from midline). A second possibility, derived from the DFT, was that shifting attention leads to systematic distortions in SWM that depend on the direction of the attention shift. In this case, we should see a systematic shift in directional errors across shifting-attention conditions, but no increase in the overall variance. Note that it was also possible that we would see a systematic shift in directional errors together with an increase in variance. This would occur if, for example, shifting spatial attention induced a bias in SWM, and also destabilized the SWM peak on some trials. Moreover, we expected to see drift away from midline, given the pervasiveness of reference-related biases in spatial-recall tasks. The critical question, however, was how such errors would be influenced by the attention manipulation. 

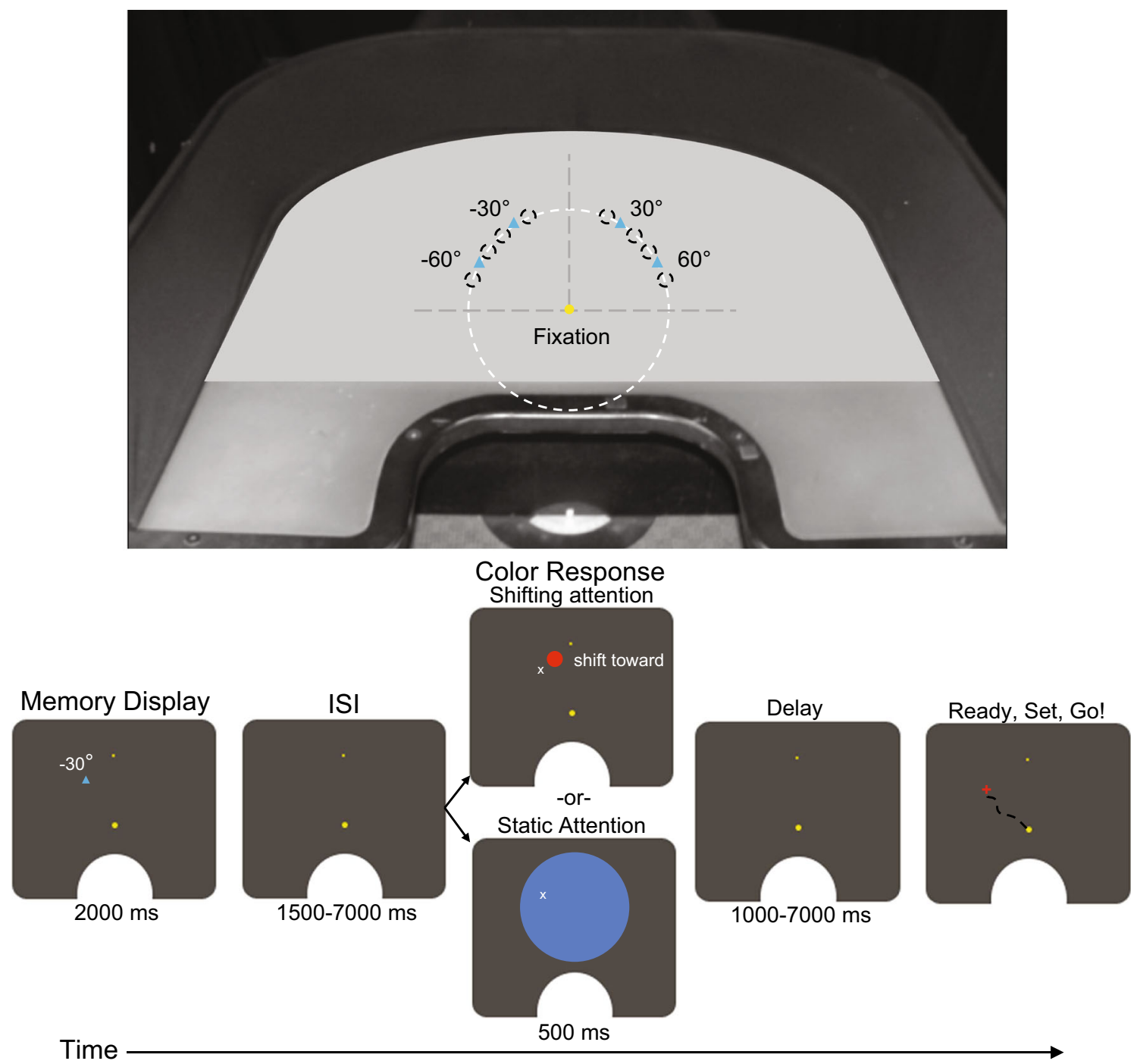

Fig. 5 Stimuli and procedures used in the behavioral experiment. Participants performed a color discrimination task interposed during the delay interval of a spatial-recall working memory task. In the shiftingattention task, performance of the task required a shift of attention either toward or away from midline, relative to the location of a remembered visual target. In the static-attention task, color discrimination could be completed without shifting attention away from the remembered location. Participants also completed control versions of these tasks, in which all of the same events occurred, but participants were not required to perform the color discrimination during the delay

\section{Method}

Participants A group of 24 University of Iowa undergraduate volunteers participated in this experiment (14 females and ten males; mean age $=20.33$ years, $S D=2.51$ ). Participants received class credit or monetary compensation $(\$ 7 / \mathrm{h})$ for their participation. All participants reported normal or corrected-tonormal visual acuity and normal color vision. One participant was replaced due to difficulties with the eyetracker.

Apparatus Throughout the session, participants were seated on a chair placed within an arc cut out from the side of a large $(0.921 \times 1.194 \mathrm{~m})$ table (see Fig. 5). The surface of the table was homogeneous, and three edges of the table were covered with a curved border to occlude the corners from participants' view. Sessions were conducted in a dimly lit room with black curtains covering the walls and ceiling. Stimuli were projected onto the surface of the table (a rear projection surface) from below using a Barco 708 data projector, with a screen resolution of $1,024 \times 768$ pixels. Responses were generated using a Microsoft Sidewinder force-feedback joystick positioned immediately to the right of the participant on the surface of the table. Stimulus presentation and response recording were controlled by a Pentium IV PC-based computer running custom software in Microsoft Visual Studio 6. Eye position data were acquired using an ISCAN RK-726 PCI head-mounted eyetracker. 
Stimuli The stimuli were presented against a gray background on the surface of the display table. Throughout each experimental trial, participants maintained fixation on a small $\left(1.66^{\circ}\right.$ in diameter ${ }^{2}$ ), yellow fixation dot positioned $21.17 \mathrm{~cm}$ in front of them on the surface of the display table $(x=512$ pixels, $y=$ 544 pixels). The memory stimuli consisted of small $\left(1.0^{\circ}\right.$ at base), light-blue equilateral triangles ("spaceships") presented at different angular separations on the periphery of an imaginary circle (radius $=10 \mathrm{~cm}$ ) centered at fixation. The sample arrays consisted of a single memory stimulus presented at one of four possible target locations, either $\pm 30^{\circ}$ or $\pm 60^{\circ}$ of angular rotation away from the midline of the display table (i.e., to the left $[-]$ or right $[+]$ of midline $=0^{\circ}$; see Fig. 5).

As in Awh et al. (1998), two types of color stimuli were presented in the color discrimination task that was completed during the retention interval of the memory task: (a) a small, colored circle subtending $1.74^{\circ}$ of visual angle, and (b) a large, colored circle subtending $17.38^{\circ}$ of visual angle. In both cases, the colors of these stimuli could be either of two different shades of blue or two different shades of red. For the small colored circles, the RGB values of the colors used were ${ }^{3}$ blue 1 $=[0,0,255]$, blue $2=[173,216,230] ; \operatorname{red} 1=[255,0,0]$, red 2 $=[255,0,255]$. For the large colored circles, the colors were blue $1=[0,0,255]$, blue $2=[25,160,235]$; red $1=[255,34,0]$, $\operatorname{red} 2=[255,70,128]$. Different color sets were used in an attempt to more closely match the color discrimination performance across conditions. When large colored circles were presented, they were always centered at fixation. Conversely, on half of the trials, the small colored circle was presented at a location $10^{\circ}$ of angular rotation closer to midline than the position of the memory stimulus that had appeared on that trial, whereas on the other half it appeared at a location $10^{\circ}$ in the opposite direction (i.e., away from midline). For example, if the memory stimulus appeared at $-30^{\circ}$ (i.e., $30^{\circ}$ to the left of midline), the small colored circle could appear at either $-20^{\circ}$ (toward midline) or $-40^{\circ}$ (away from midline).

Design We used a within-subjects design with four different experimental conditions: a dual-task shifting-attention condition,

\footnotetext{
${ }^{2}$ Visual angles were estimated by using the distance of the stimulus from the front edge of the table and assuming an eye height of $42 \mathrm{~cm}$ above the table surface. These values are approximately correct when head position is fixed using a chinrest attached to the front edge of the table. However, because head position was not fixed during the experimental session, the exact distance of each participant from the location of the stimuli on the table and the height of the eye above the table likely differed to some extent across participants, as well as for the same participant at different points during the experimental session.

${ }^{3}$ Because the display surface used in the present experiment was a rear projection screen, it was difficult to obtain accurate color coordinates (e.g., CIE $x, y$, and luminance) using the colorimeter we had available. Therefore, we report RGB values. Additionally, the color values reported for the color blue 2 in the small-colored-circle task are approximate values based on the stimuli used during pilot testing. The precise final values were not available, due to a hard-drive failure.
}

in which the small-colored-circle color discrimination task was completed during the delay interval of the spatial-recall task; a dual-task static-attention condition, in which the large-coloredcircle discrimination task was completed during the delay interval; and two single-task control conditions that were identical to the shifting-attention and static-attention conditions, with the exception that the color discrimination task was not completed during the delay interval. There were two independent variables in the shifting-attention conditions: the location of the memory target $\left( \pm 30^{\circ}, \pm 60^{\circ}\right)$ and the direction of the color discrimination stimulus (toward vs. away from midline).

Procedures At the beginning of the experimental session, the experimenter explained the task and showed the participant a demonstration of each condition. The sequence of events in a trial is depicted at the bottom of Fig. 5. Each trial began with the appearance of a yellow "start dot" and a set of green crosshairs that could be moved using the joystick. When the dot appeared, participants were instructed to move the crosshairs to the dot's position and to fixate the dot throughout the subsequent trial. If the eyes moved off of the start dot at any point during the trial, the trial was halted, the participant was prompted to return to the start location, and the trial was repeated.

Once the crosshairs and the eyes were positioned over the start dot, a stimulus was presented for $2,000 \mathrm{~ms}$ at one of the four possible target locations $\left( \pm 30^{\circ}, \pm 60^{\circ}\right)$. This was followed by an ISI that varied randomly from 1,500 to $7,000 \mathrm{~ms}$, and a 500 -ms presentation of either a small or a large colored circle, depending on the experimental condition. When the colored circle appeared during dual-task trials, participants made a speeded discrimination response, indicating which of two possible colors they saw by pressing one of two buttons on the base of the joystick using the middle and index fingers of the left hand. This was immediately followed by auditory feedback, in the form of a laser sound for correct responses, or a buzzer sound for incorrect responses. Participants also received auditory feedback if they failed to respond to the discrimination target within $1,000 \mathrm{~ms}$ of stimulus offset. The offset of the colored circle was followed by a variable delay of 1,000-7,000 ms, at which point the participant heard "ready, set, go," with the word "go" occurring exactly $10 \mathrm{~s}$ after the offset of the original memory display. On "go," participants moved the crosshairs to the remembered spaceship location with their right hand using the joystick, and clicked the trigger on the joystick using the right index finger. Once they had made their recall response, the spaceship stimulus reappeared for $500 \mathrm{~ms}$, followed by a $1,500-\mathrm{ms}$ feedback screen that gave detailed information about their performance on that trial (see the details in Spencer \& Hund, 2002). The next trial began after a 1,000-ms intertrial interval.

The experiment began with a series of single-task practice blocks designed to familiarize participants with the tasks. First, they completed 12 trials of the spatial memory task by itself: 
three trials for each of the four memory targets. This was followed by eight trials each of the small-colored-circle and large-colored-circle tasks alone. Additionally, participants completed a short practice block at the beginning of each experimental block during the session. For the shifting-attention condition and its corresponding control condition, the practice blocks consisted of a total of eight trials: two trials for each memory target (four), with the color (e.g., blue1 or blue2) and direction (toward vs. away from midline) of the small colored circle occurring with equal likelihoods. For the static-attention condition and its corresponding control condition, participants completed four practice trials: one trial for each memory target (four), with each color occurring with equal likelihood. This resulted in a total of 24 regular practice trials.

For the shifting-attention and -control conditions, the experimental blocks consisted of 32 trials: four trials for each memory target (four), with the color discrimination target appearing in each direction (two) and with color counterbalanced across trials. For the static-attention and -control conditions, participants completed 16 trials: four trials for each target (four), with each color appearing equally often. Thus, participants completed a total of 148 trials during the experimental session: 28 single-task practice trials at the beginning of the session, 24 regular practice trials, and 96 total experimental trials. The color sets used in the discrimination task were counterbalanced across participants, with half of the participants responding to the red color set and the other half to the blue set. For the control conditions, each participant saw the opposite color set. Conditions were blocked, and they were counterbalanced across participants using a balanced Latin square design.

Method of analysis To calculate the spatial-recall errors for each trial, we recorded the $x$ - and $y$-coordinates of the crosshairs when the participant pulled the joystick trigger. Using these values, we calculated three separate measures of error: (1) directional error, which was computed as the angular separation between the vector from the start location to the response and the vector from the start location to the actual target location (in degrees); (2) variable error, which reflected the variation in directional errors across trials; and (3) extent error, which corresponded to the difference between the lengths of the vector from the start location to the location of the response and the vector from the start location to the actual target location, giving an indication of the extent to which the recall responses over- or undershot the target location. Directional errors were computed such that positive errors were in a direction away from the midline symmetry axis of the table, and negative errors reflected errors toward midline. The directional and extent errors were checked for outliers by removing trials on which errors were greater than \pm 2 standard deviations from the mean error for a given participant. This resulted in the removal of $<4 \%$ of all trials. The mean directional and extent errors were computed by averaging across trials to the same target and, for the shifting-attention conditions, the same direction of the attentional shift. Prior to conducting our statistical analyses, we collapsed across targets positioned the same distance from midline on the left and right sides of the screen (i.e., across $\pm 30^{\circ}$ and $\pm 60^{\circ}$ targets). We also calculated percent correct accuracy as a measure of performance in the color discrimination task.

\section{Results and discussion}

Shifting-attention and -control conditions Mean directional errors for the shifting-attention and shifting-control conditions are shown in Fig. 3B. As expected, participants' estimation responses revealed substantial drift away from the midline of the task space (positive errors), and larger errors for targets near versus far from midline. In keeping with the findings from the simulation experiment, participants made substantially larger errors when the small colored circle was presented in the direction away from versus toward midline. Importantly, this difference was roughly three times larger in the shifting-attention condition (mean difference $=2.83^{\circ}$ ), in which participants were required to complete the color discrimination task, than in the shifting-control condition (mean difference $=1.0^{\circ}$ ), in which they were instructed to ignore the color stimuli. This finding suggests that memory for location was attracted toward the focus of attention when attention was shifted to the location of the color stimulus to complete the discrimination task during the delay.

Static-attention and -control conditions Mean directional errors in the static-attention and static-control conditions are shown in Fig. 3C. As was seen in the shifting-attention and -control conditions, participants' estimation responses revealed a systematic drift away from the midline of the table (positive errors) in each condition, with substantially larger errors for $\pm 30^{\circ}$ targets than for $\pm 60^{\circ}$ targets. Additionally, errors were larger overall in the static-attention condition than in the static-control condition; thus, there was a nonspecific increase in errors when participants had to complete the dual task. In keeping with these findings, a repeated measures ANOVA with Condition (static-attention, static-control) and Target $\left( \pm 30^{\circ}, \pm 60^{\circ}\right)$ as factors revealed significant main effects of condition, $F(1,23)=5.36, p=.03, \eta_{\mathrm{p}}{ }^{2}=.19$, and of target, $F(1,23)=48.55, p<.001, \eta_{\mathrm{p}}{ }^{2}=.68$. The interaction was not significant, $F<1$.

These data were analyzed in a three-way, repeated measures analysis of variance (ANOVA) with Condition (shifting attention, shifting control), Color Direction (toward, away), and Target $\left( \pm 30^{\circ}, \pm 60^{\circ}\right)$ as factors. This analysis revealed significant main effects of target, $F(1,23)=31.49, p<.001$, $\eta_{\mathrm{p}}{ }^{2}=.58$, and color direction, $F(1,23)=5.89, p=.023, \eta_{\mathrm{p}}{ }^{2}=$ .20. The Condition $\times$ Target interaction was also significant, $F(1,23)=4.60, p=.042, \eta_{\mathrm{p}}{ }^{2}=.17$, as was the Condition $\times$ 
Color Direction interaction, $F(1,23)=4.63, p=.042, \eta_{\mathrm{p}}{ }^{2}=$ .17. All additional effects were not significant (all $p \mathrm{~s}>.20$ ).

The significant Condition $\times$ Target interaction was analyzed using tests of simple effects comparing the mean directional errors in the shifting-attention and shifting-control conditions at each target location. This analysis revealed no significant effect of attention condition at either the $30^{\circ}$ or the $60^{\circ}$ target locations, all $p \mathrm{~s}>.11$, although errors were approximately $1^{\circ}$ larger overall to targets in the control condition. Additionally, we compared mean directional errors across targets for the shifting-attention and shifting-control conditions separately. This analysis revealed significant main effects of target for both conditions, $F_{\mathrm{s}}(1,23)>15.0$, all $p \mathrm{~s}<.001, \eta_{\mathrm{p}}{ }^{2} \mathrm{~s}$ $\geq .39$, although the slope across targets was steeper in the shifting-attention condition (see Fig. 3B).

More central to our experimental hypotheses was the significant Condition $\times$ Color Direction interaction. To explore this interaction, we again conducted tests of simple effects looking at errors when the color target appeared toward versus away from midline in the shifting-attention and -control conditions separately. If increased errors in the shift-away versus the shift-toward condition reflect the voluntary shifting of attention to the location of the color stimulus, rather than stimulus-driven orienting to the location of a sudden onset, we would expect to find a significant effect of color direction in the shifting-attention, but not the shifting-control, condition. Consistent with this proposal, these analyses revealed a significant main effect of color direction for the shiftingattention condition, $t(23)=2.92, p<.01$, Cohen's $d_{z}=$ 0.596 , but not for the shifting-control condition, $p>.20$. This observation runs contrary to the findings of Van der Stigchel et al. (2007), discussed above. Note, however, that given the relatively small sample size of the present experiment, this null effect might reflect a Type II error. Nonetheless, the present results suggest that the effects of color direction observed in the shifting-attention condition were substantially larger than the small modulation observed in its control condition.

In addition to the analyses of directional errors, separate three-way ANOVAs were conducted to examine differences in extent errors and the variability of directional errors. The analysis of variable error revealed a significant Condition $\times$ Target interaction, $F(1,23)=10.37, p=.004, \eta_{\mathrm{p}}{ }^{2}=.31$, with greater overall variable error for the $\pm 60^{\circ}$ targets in the shiftingattention condition than in its control condition. The Condition $\times$ Color Direction interaction was not significant, nor were any other main effects or interactions (all $p \mathrm{~s}>.22$, with the exception of the main effect of color direction, for which $p=.11$ ). The analysis of extent errors revealed no significant main effects or interactions, although the main effect of target approached significance $(p=.06$; all other $p \mathrm{~s}>.1)$.

Color discrimination Performance in the color discrimination task is shown in Fig. 3D. Color discrimination performance was uniformly high overall, but was somewhat better for the small-colored-circle task than for the largecolored-circle task. This was confirmed by a one-way ANOVA showing a trend toward a main effect of condition, $F(1,23)=4.15, p=.053$. This result contrasts with the findings of Awh et al. (1998, Exp. 3), in which performance was found to be worse in the small- than in the large-colored-circle task. This discrepancy likely reflects differences in the particular colors used in the color discrimination tasks across studies. In particular, for our experiment the colors used in the small-colored-circle task were highly discriminable (e.g., blue and light-blue), whereas in the large-colored-circle task, the differences between colors were more subtle (e.g., two similar shades of blue). Although our results contrast with those of Awh et al., the relative difficulty of the large-colored-circle task ensured that any increase in errors found in the shiftingversus the static-attention conditions cannot be attributed to differences in the overall difficulty of the color discrimination task in this condition.

In summary, consistent with simulations of the DFT, our behavioral results revealed that memory for location was attracted toward the focus of attention when attention was shifted to a nearby, nonmemorized location during the delay interval of a spatial-recall task. Although qualitatively similar differences were observed in the shifting-control conditions, these differences did not reach statistical significance. Interestingly, we also observed an increase in variable errors for the $\pm 60^{\circ}$ targets in the shifting-attention versus -control conditions. This would be expected if participants were simply forgetting the target location and randomly guessing on a larger proportion of shifting-attention than of shifting-control trials for these targets. Why these effects were only observed for the $\pm 60^{\circ}$ targets is unclear.

Taken together, these results suggest that shifting attention modulates the patterns of delay-dependent spatial biases seen in spatial-recall tasks. What does this indicate about the role of spatial attention in SWM? The finding of systematic shifts in SWM as a function of attention is consistent with the DFT and the specific modulatory role of attention in the model. Is attention also required for maintenance? Although the increase in variances observed for the $\pm 60^{\circ}$ targets is consistent with this proposal, the systematic bias in SWM was not. Moreover, if attention were directly responsible for maintenance in SWM, we would expect variance to be affected irrespective of the target location. A more plausible interpretation, therefore, is that spatial attention serves to modulate the patterns of activation in SWM, but is not the rehearsal mechanism itself.

\section{General discussion}

In the present study, we adapted a dynamic-field model of SWM to capture performance in a recall variant of the shifting-attention 
condition of Awh et al. (1998, Exp. 3), in which spatial discrimination was found to be impaired when attention was shifted to discriminate a color target during the delay. The model consisted of a three-layer neural-field model of SWM, which was initially developed to capture performance in spatial recall, reciprocally coupled to a 2-D CS field that implemented a simple form of color discrimination. Using the model, we simulated performance in a shifting-attention condition, in which a color discrimination task was performed during the delay period of a spatialrecall task. Importantly, the color discrimination target could appear either toward or away from a midline reference axis, relative to the memorized location. We hypothesized that weak excitatory input from the CS field to the SWM layer of the threelayer model at the location of the discrimination target would modulate the patterns of delay-dependent drift away from midline, increasing drift when the colored stimulus appeared away from midline, and decreasing drift when the stimulus was presented toward midline relative to the memory target. As is shown in Fig. 3A, the simulation results supported this proposal: Drift away from midline was found to be substantially larger when attention was shifted away from versus toward midline. A qualitatively similar, though much smaller, pattern of modulation was also observed in a shifting-control condition, in which the color stimuli appeared but failed to induce a robust peak in the CS field (see Fig. 2D).

Critically, a similar pattern of results was observed in a behavioral experiment that tested the model's predictions. Errors were nearly $3.0^{\circ}$ larger when attention was shifted away from rather than toward midline during the delay, as compared to a nonsignificant $1.0^{\circ}$ difference in a control condition in which attention did not need to be shifted away from the memorized location (Fig. 3B). Additionally, we also observed a difference in the overall variance of recall responses in the shifting-attention and -control conditions, but this effect was only observed for the $\pm 60^{\circ}$ targets.

Taken together, these results suggest that the decrement in delayed spatial discrimination performance observed by Awh et al. (1998) may have been caused by attention-related distortions of SWM representations, rather than by their failure. This explanation is at odds with the proposal that continued spatial attention is required for the successful maintenance of location information in SWM. Recall that maintenance (i.e., self-sustained activation) in the model emerges through interactions between excitatory and inhibitory neural populations that respond selectively to spatial location. Although the stability and precision of maintained patterns of activation can be influenced by additional factors (e.g., the presence of reference frames, inputs from memory traces, attention, etc.), they do not strictly depend on these factors.

Although our account is at odds with the proposal from Awh and colleagues (1998), there are also points of agreement. Specifically, we are in agreement that attention and working memory share overlapping mechanisms. Indeed, in many cases it can be difficult to determine where working memory ends and attention begins. For example, the local-excitation/lateral-inhibition function that underlies maintenance in the model also implements one of the core features of selective attention: the selection of one or more targets or locations out of potentially many possibilities. Specifically, at the limit, when lateral inhibition is broad and relatively strong, the model functions as a winner-takes-all network, building stable peaks of activation in response to only the most salient target inputs, and suppressing activation associated with other targets. When the model is functioning in this mode, the relative salience of a given target is determined by a combination of its visible properties (e.g., how similar it is to the other targets in the array) and top-down factors reflecting the relevance of the target or one or more of its features to the task at hand, as in other prominent models of attention (Deco \& Rolls, 2004; Mozer \& Sitton, 1998; Wolfe, 1994). Thus, prior to peak formation, interactions within the SWM field can serve an attentional function, whereas once a stable peak of activation is formed, the same interactions support sustained activation, which is a core feature of working memory.

Another form of attention in the model, which can also influence maintenance in working memory, is top-down "preshape" input to the CS field in the shifting- and staticattention conditions. Such inputs capture the requirements of the color discrimination task that participants completed during the delay interval of the spatial-recall task. Specifically, this input produced a spatially global baseline shift in the firing rates of neurons in the CS field tuned to the colors used in the task, in keeping with studies of feature-based attention (see, e.g., Liu \& Mance, 2011; Maunsell \& Treue, 2006; Serences \& Boynton, 2007). This allowed a peak of activation to be created following stimulus presentation and maintained in the CS field long enough for a response to be generated. Thus, in addition to ensuring that an above-threshold peak of activation was present in the CS field only when a taskrelevant color was presented to the model, preshape inputs also made it possible for the color and location of the color stimulus to be retained for a brief time. Although the sources of such "preshaping" inputs are not specified in the model, a reasonable possibility is that they reflect top-down inputs from other neural populations responsible for maintaining taskspecific information in an active state, as was proposed by Liu and colleagues (Liu, Slotnick, Serences, \& Yantis, 2003) and is captured in several other models of attention (Desimone \& Duncan, 1995; Duncan \& Humphreys, 1989; Mozer \& Sitton, 1998). Interestingly, this simple form of feature-based attention, rather than a specifically spatial form of attention, plays a key role in allowing us to capture the pattern of spatial biases reported here.

This brings us to another closely related form of attention at work in the model: modulations of patterns of activation in one population of neurons, via inputs from another population to which it is coupled. For example, modulations of peak drift in 
the model reported here arise as a result of input from a spatially organized population of neurons that maintain color information for use in the color discrimination task. Similarly, excitatory feedback from SWM to this field allowed us to capture the RT advantages reported in Experiment 1 of Awh et al. (1998). Thus, the key pattern of results here and in the experiments of Awh and colleagues (1998) can be viewed as emerging from the reciprocal interactions of ventral- and dorsal-stream neural populations that specialize in primarily visual and primarily spatial forms of information, respectively (Goodale \& Milner, 1992; Ungerleider $\&$ Mishkin, 1982). Although these systems are semi-independent, evidence of substantial crosstalk between them has been reported. For example, Claeys and colleagues (2004) showed that performance of color discrimination tasks involves interactions between ventral-stream areas that respond selectively to color and dorsal-stream areas that may play a role in selecting an appropriate response. Additionally, Zhong and Rockland (2003) reported evidence of spatially specific projections from regions of the parietal cortex to anterior portions of area TE in the macaque, a canonical ventral-stream area. Moreover, inputs from oculomotor structures such as the frontal eye fields (FEF) and portions of the parietal cortex have been proposed as likely sources of spatial-reentry signals that could serve as attentional feedback to ventral-stream areas such as V4 (Hamker, 2005).

Finally, a variant of attention can also be realized through global modulations of the resting levels of neurons within a field. For instance, in the color discrimination task reported here, the resting level in the CS field was set so that peaks were only possible when a color matching one of the color targets was present in the task space (i.e., when a color appeared at a preshaped site in the field). This prevented the model from building a peak in the CS field in response to the blue "spaceship" memory target, or in response to the color stimuli in each of the control conditions (i.e., to task-irrelevant stimuli). More generally, modulations of the resting level within a given field can make the formation of peaks either more or less likely, and once a peak has formed, either easier or harder to stabilize. A similar mechanism was implemented in a model of SWM proposed by Edin and colleagues (2009; see also Roggeman, Klingberg, Feenstra, Compte, \& Almeida, 2014) as a means of controlling the capacity of SWM. In their model, a nonspecific "boost" input from a putative frontal control area increased the number of distinct peaks of activation that could be sustained in a posterior "storage network." Thus, global modulations of resting level can also play a role in the creation and maintenance of self-sustained patterns of activation in working memory - a key function of attention.

The different varieties of attention discussed above lead naturally to a critical question: Where precisely is spatial attention in our model? The answer is that spatial attention is not isolated to one mechanism; rather, varieties of spatial attention are realized via different functionalities of the model within a task-specific context. This differs from the common conception of spatial attention as a type of mental spotlight that can be focused on different locations, more or less independently of the objects occupying those locations (for critiques of this conceptualization of attention, see Driver \& Baylis, 1989; Huang \& Pashler, 2007; LaBerge \& Brown, 1989; LaBerge, Carlson, Williams, \& Bunney, 1997). Given that the attentional effects captured by our model emerged as the model processed localized inputs, this common perspective of attention raises a key issue: Is there some type of spotlight in our model?

Simulations of Experiment 1 of Awh et al. (1998; see Fig. 4) illustrate one type of spotlight effect in our model. In this task, attention was initially cued to a particular location by the appearance of a stimulus at a to-be-remembered location. This was followed by a discrimination decision that was enhanced at the remembered location. Thus, one could say that the spotlight of attention was fixed at the remembered location. Consistent with this, our model showed faster discrimination when targets were presented at the to-be-remembered location (Fig. 4D). Analysis of our model, however, shows that such effects do not require an attention system that is distinct from the processes engaged in the sustained maintenance of locations in SWM. That is, holding a location in SWM effectively modulates activation in coupled cortical areas, giving a competitive advantage to targets appearing at the memorized location. This is consistent with behavioral and neuroimaging studies of working memory and attention (Awh et al., 2000; Awh et al., 1999; Hillyard \& Anllo-Vento, 1998; Hillyard et al., 1998; Postle et al., 1999). Thus, although our model shows spotlight-like effects, such effects do not necessarily reflect distinctly "attentional" mechanisms.

\section{Relationship of the DFT to other models of attention and working memory}

The dynamic-field model reported here shares many features with other prominent models of attention and working memory. For example, similar to the neurodynamical models proposed by Deco and Rolls (2004) and Hamker (2005), there is no dedicated "attention field" or stack of fields that uniquely instantiate spatial attention. Rather, attention-like phenomena arise as emergent products of reciprocal coupling among neural populations specialized for different functions, with inputs from one neural population modulating the local intracortical dynamics of the other (see also Desimone \& Duncan, 1995; Duncan, Humphreys, \& Ward, 1997; and the discussion of attention in Fuster, 2003). For example, in Hamker's model, spatially specific activation in oculomotor structures such as the FEF, which reflects the locations of potential saccade targets, serves as an attentional reentry signal to spatially organized ventral-stream areas such as V4. Top-down inputs from the ventrolateral portion of the prefrontal cortex, which is implicated in SWM, can influence saccade plans in the FEF, allowing a spatial focus of attention reflecting the likely location of a 
subsequent stimulus to affect early visual processing. Similarly, in the model of Deco and Rolls, feedback from prefrontal areas implicated in working memory for stimulus features (e.g., color or shape) to spatially organized populations of neurons in the ventral stream (e.g., V4) allow neural resources to be focused on locations containing those features. The model proposed here is clearly in line with these neurodynamical approaches to attention and working memory.

Models such as these have been contrasted with the "classical" view of attention, wherein an initial parallel stage of processing is followed by a serial, focal attention stage that results in the serial selection of targets mediated by spatial location (see, e.g., Neisser, 1967; Treisman \& Gelade, 1980; Wolfe, 1994). Spatial attention is typically implemented in such models as a dedicated "saliency map" or "attentional map," which tags specific locations in the visual field as being noteworthy on the basis of top-down and bottom-up information about the stimuli present (for a formal implementation of the saliency map idea, see Itti \& Koch, 2001; Mozer \& Sitton, 1998). Although no such dedicated attentional map need be invoked to explain the present findings, something similar could be implemented in our model through the inclusion of an attention or fixation system that gates inputs to the perceptual layer of the three-layer working memory model that are outside the currently fixated (or attended) region of the visual field, similar to the model of Hamker (2005) discussed above. Indeed, Schneegans and colleagues (Schneegans, Spencer, Schöner, Hwang, \& Hollingworth, 2014) recently proposed a neural-field model that captures dynamic interactions between visual working memory and saccade target selection, which could be incorporated in the model proposed here (for an early precursor to this model, see Kopecz \& Schöner, 1995).

Finally, with regard to working memory, the DFT is in a class of neural-network models that achieves a stable memory state using bistable networks in which a stable state of sustained activation coexists with an "off" state (Amari, 1977; Amari \& Arbib, 1977; Compte et al., 2000; Edin et al., 2009; Wei, Wang, \& Wang, 2012). Such models are consistent with the now-classical view that stimulus-specific information is maintained over brief temporal intervals via the sustained activation of feature-selective populations of neurons (for an alternative to sustained-activation-based forms of maintenance, see Riggall \& Postle, 2012).

\section{Conclusions}

In the present study, we used a combination of behavioral experimentation and formal modeling to investigate the interactions between spatial attention and SWM. Specifically, we used a neural model to simulate performance in a task that involved performance of a color discrimination task interposed during the delay interval of a spatial-recall task. Previous evidence from spatial delayed recognition has suggested that shifting attention to the location of the discrimination target during the delay leads to the failure of working memory representations, and a consequent decline in performance. Contrary to this proposal, the model simulations and behavioral findings reported here suggest that SWM is biased toward the focus of attention, which suggests that the increased errors observed in previous studies may have been due to systematic distortions of the SWM representations, rather than to their failure. Further consideration of the model's functioning suggested the presence of multiple overlapping mechanisms of attention and working memory. Although each of the mechanisms discussed above plays a role in generating the phenomena that have been traditionally identified as reflecting the operation of attention or working memory, importantly, none of them map directly onto these psychological constructs in a straightforward manner. This highlights the need to move the investigation of these constructs to a more formal level. In the present report, we contribute to this goal, moving in the direction of neurocomputational modeling that will allow us to investigate neural mechanisms operating at the population level while retaining strong links to behavioral experimentation.

Author note This research was made possible by National Institute of Health Grant No. RO1 MH62480, and by National Science Foundation Grant Nos. BCS 00-91757 and HSD 0527698 awarded to J.P.S.

\section{Appendix: Model equations}

In this appendix, we describe the equations that govern activation in each layer of the three-layer model and the CS field used in the present report. Although the equations share most components, we describe each separately to highlight the unique contributions to the dynamics of each layer.

Activation in the perceptual field, $\operatorname{PF}(u)$, is captured by

$$
\begin{aligned}
& \tau \dot{u}(x, t)=-u(x, t)+h_{u}+\int c_{u u}\left(x-x^{\prime}\right) \Lambda_{u u}\left(u\left(x^{\prime}, t\right)\right) d x^{\prime} \\
&-\int c_{u v}\left(x-x^{\prime}\right) \Lambda_{u v}\left(v\left(x^{\prime}, t\right)\right) d x^{\prime} \\
&+s_{\text {ref }}(x, t)+s_{\text {tar } r_{\text {space }}}(x, t)+s_{\text {tar }}(x \text { olor } \\
&(x, t)
\end{aligned}
$$

where $\dot{u}(x, t)$ is the rate of change of the activation level for each neuron across the spatial dimension, $x$, as a function of time, $t$. The constant $\tau$ determines the time scale of the dynamics (Erlhagen \& Schöner, 2002). The first factor that contributes to the rate of change of activation in PF is the current activation in the field, $-u(x, t)$, at each site $x$. This component is negative so that activation changes in the direction of the resting level $h_{u}$.

Next, activation in PF is influenced by the local-excitation/ lateral-inhibition interaction profile, defined by self-excitatory projections, $\int c_{u u}\left(x-x^{\prime}\right) \Lambda_{u u}\left(u\left(x^{\prime}, t\right)\right) d x^{\prime}$, and inhibitory projections from the inhibitory layer (Inhib; $v), \int_{c_{u v}}\left(x-x^{\prime}\right) \Lambda_{u v}\left(v\left(x^{\prime}\right.\right.$, 
t)) $d x^{\prime}$. Thessssse projections are defined by the convolution of a Gaussian kernel with a sigmoidal threshold function. In particular, the Gaussian kernel was specified by

$c\left(x-x^{\prime}\right)=c \exp \left[-\frac{\left(x-x^{\prime}\right)^{2}}{2 \sigma}\right]-k$,

with strength $c$, width $\sigma$, and resting level $k$. The sigmoidal function is given by

$\Lambda(u)=\frac{1}{1+\exp [-\beta u]}$,

where $\beta$ is the slope of the sigmoid - that is, the degree to which the neurons close to threshold (i.e., 0) contribute to the activation dynamics. Lower slope values permit graded activation near threshold to influence performance, whereas higher slope values ensure that only above-threshold activation contributes to the activation dynamics. At extreme slope values, the sigmoid function approaches a step function.

Finally, in the full version of the model, $\mathrm{PF}$ receives input from the world via a second perceptual field in a retinal frame of reference (Spencer et al., 2007). For simplicity, in the present simulations we replaced this retinal field with direct input already in an objectcentered reference frame. In particular, two inputs passed activation to PF: the reference input (e.g., perception of the midline axis in the recall task) and target input (e.g., the transient presentation of a target object in the task space). All inputs took the form of a Gaussian,

$$
\begin{aligned}
& S_{\text {tar } r_{\text {space }}}(x, t)=c \exp \left[-\frac{\left(x-x_{\text {center }}\right)^{2}}{2 \sigma^{2}}\right] \chi^{(t)}, S_{\text {tar }_{\text {color }}}(x, t) \\
& \quad=c \exp \left[-\frac{\left(x-x_{\text {center }}\right)^{2}}{2 \sigma^{2}}\right] \chi^{(t)}
\end{aligned}
$$

centered at $x_{\text {center }}$, with width $\sigma$ and strength $c$. These inputs could be turned on and off through time (e.g., the target appears and then disappears). This time interval was specified by the step function $\chi^{(t)}$ (see the text for details on the timing of inputs).

The second layer of the model, $\operatorname{Inhib}(v)$, is specified by the following equation:

$$
\begin{aligned}
\tau \dot{v}(x, t) & =-v(x, t)+h_{v}+\int c_{v u}\left(x-x^{\prime}\right) \Lambda_{v u}\left(u\left(x^{\prime}, t\right)\right) d x^{\prime} \\
& +\int c_{v w}\left(x-x^{\prime}\right) \Lambda_{v w}\left(w\left(x^{\prime}, t\right)\right) d x^{\prime} .
\end{aligned}
$$

As before, $\dot{v}(x, t)$ specifies the rate of change of activation across the population of spatially tuned neurons, $x$, as a function of time, $t$; the constant $\tau$ sets the time scale, $v(x, t)$ captures the current activation of the field, and $h_{v}$ sets the resting level of neurons in the field. Note that Inhib receives activation from two projections - one from PF, $\int_{c_{v u}}\left(x-x^{\prime}\right) \Lambda_{v u}\left(u\left(x^{\prime}\right.\right.$, $t)) d x^{\prime}$, and one from SWM, $\int_{\mathcal{C}_{v w}}\left(x-x^{\prime}\right) \Lambda_{v w}\left(w\left(x^{\prime}, t\right)\right) d x^{\prime}$. As was described above, these projections are defined by the convolution of a Gaussian kernel (Eq. 2) with a sigmoidal threshold function (Eq. 3).

The third layer, $\operatorname{SWM}(w)$, is governed by the following equation:

$$
\begin{aligned}
& \tau \dot{w}(x, t)=-w(x, t)+h_{w}+\int c_{w w}\left(x-x^{\prime}\right) \Lambda_{w w}\left(w\left(x^{\prime}, t\right)\right) d x^{\prime} \\
& -\int c_{w v}\left(x-x^{\prime}\right) \Lambda_{w v}\left(v\left(x^{\prime}, t\right)\right) d x^{\prime}+\int c_{w u}\left(x-x^{\prime}\right) \Lambda_{w u}\left(u\left(x^{\prime}, t\right)\right) d x^{\prime} \\
& +\int c_{w w_{c, s}}\left(x-x^{\prime}\right) \Lambda_{w w_{c, s}}\left(w_{c, s}\left(x^{\prime}, t\right)\right) d x^{\prime}+c_{s} s_{r e f}(x, t)+c_{S} s_{t a r_{c o l o r}}(x, t) .
\end{aligned}
$$

Again, $\dot{w}(x, t)$ is the rate of change of activation across the population of spatially tuned neurons, $x$, as a function of time, $t$; the constant $\tau$ sets the time scale, $w(x, t)$ captures the current activation of the field, and $h_{w}$ sets the resting level. SWM receives self-excitation, $\int_{c_{w w}}\left(x-x^{\prime}\right) \Lambda_{w w}\left(w\left(x^{\prime}, t\right)\right) d x^{\prime}$, lateral inhibition from $\operatorname{Inhib}(v), \int_{c_{w v}}\left(x-x^{\prime}\right) \Lambda_{w v}\left(v\left(x^{\prime}, t\right)\right) d x^{\prime}$, and input from $\operatorname{PF}(u), \int_{c_{w u}}\left(x-x^{\prime}\right) \Lambda_{w u}\left(u\left(x^{\prime}, t\right)\right) d x^{\prime}$. SWM also receives input from the spatial dimension of a single CS field $\left(w_{c, s}\right)$, $\int_{c_{w w_{c, s}}}\left(x-x^{\prime}\right) \Lambda_{w w_{c, s}}\left(w_{c, s}\left(x^{\prime}, t\right)\right) d x^{\prime}$, and direct reference, $S_{r e f}(x$, $t$ ), and target, $S_{\text {tar }}(x, t)$, inputs scaled by $c_{s}$.

Finally, for the CS field ( $w_{\text {colorspace }}$ ), the basic concepts of the field dynamics and the mechanisms that support the maintenance of localized peaks of activation were generalized to two dimensions (i.e., color and space; see Erlhagen \& Schöner, 2002). The dynamical equations governing activation in the CS field have the same general form as in Eqs. 1, 5, and 6 above:

$$
\begin{aligned}
& \tau \dot{w}_{\text {color }, \text { space }}(x, y, t)=-w_{c, s}(x, y, t)+h_{w_{c, s}} \\
& \quad+\iint c_{w_{c, s} w_{c, s}}\left(x-x^{\prime}, y-y^{\prime}\right) \Lambda_{w_{c, s} w_{c, s}}\left(w_{c, s}\left(x^{\prime}, y^{\prime}, t\right)\right) d x^{\prime} d y^{\prime} \\
& \quad+\int c_{w_{s} w}\left(x-x^{\prime}\right) \Lambda_{w_{s} w}\left(w\left(x^{\prime}, t\right)\right) d x^{\prime} \\
& \quad+s_{\text {pre }}(y, t)+s_{\text {tar }}(x p a c e \\
&
\end{aligned}
$$

As before, $\dot{w}_{\text {color,space }}(x, y, t)$ is the rate of change of activation across the population of spatially tuned neurons, $x$, and the population of color-tuned neurons, $y$, as a function of time, $t$; the constant $\tau$ sets the time scale; $w_{c, s}(x, y$, $t$ ) captures the current activation of the field; and $h_{w c, s}$ sets the resting level. For this field, the local-excitation/lateralinhibition form of interaction is captured by a single equation, $\iint c_{w_{c, s} w_{c, s}}\left(x-x^{\prime}, y-y^{\prime}\right) \Lambda_{w_{c, s} w_{c}}\left(w_{c, s}\left(x^{\prime}, y^{\prime}, t\right)\right) d x^{\prime} d y^{\prime}$. In contrast to the three-layer model, in which inhibition is mediated by a separate field of inhibitory neurons, both the excitatory and inhibitory components of the interaction 
are defined by the convolution of a Gaussian kernel with a sigmoidal threshold function. Specifically, the 2-D Gaussian kernel was specified by

$$
\begin{gathered}
c\left(x-x^{\prime}, y-y^{\prime}\right)=c_{\text {excite }, x} \exp \left[-\frac{\left(x-x^{\prime}\right)^{2}}{2 \sigma^{2}}\right]+c_{\text {excite }, y} \exp \left[-\frac{\left(y-y^{\prime}\right)^{2}}{2 \sigma^{2}}\right] \\
-c_{\text {inhib }, x} \exp \left[-\frac{\left(x-x^{\prime}\right)^{2}}{2 \sigma}\right]-c_{\text {inhib }, y} \exp \left[-\frac{\left(y-y^{\prime}\right)^{2}}{2 \sigma^{2-}}\right]-w_{\text {inhib }, x y}
\end{gathered}
$$

with strength $c$, width $\sigma$, and an additional inhibitory term, $w_{\text {inhib,xy }}$ which provides global inhibition to the field. Finally, the CS field receives excitatory feedback from $\operatorname{SWM}(w), \int_{\mathcal{C}_{w_{s}}}\left(x-x^{\prime}\right) \Lambda_{w_{s} w}\left(w\left(x^{\prime}, t\right)\right) d x^{\prime}$, direct preshape input along the color dimension, $s_{p r e}(y, t)$, and direct target input, $s_{\text {tar }}$ space $(x, y, t)$ and $s_{\text {tar }}$ color $(x, y, t)$, reflecting the color and spatial locations of the memory target and the color target, respectively.

\section{References}

Amari, S. (1977). Dynamics of pattern formation in lateral-inhibition type neural fields. Biological Cybernetics, 27, 77-87.

Amari, S., \& Arbib, M. A. (1977). Competition and cooperation in neural nets. In J. Metzler (Ed.), Systems Neuroscience (pp. 119-165). New York, NY: Academic Press.

Awh, E., Anllo-Vento, L., \& Hillyard, S. A. (2000). The role of spatial selective attention in working memory for locations: Evidence from event-related potentials. Journal of Cognitive Neuroscience, 12, 840-847. doi:10.1162/089892900562444

Awh, E., \& Jonides, J. (1998). Spatial working memory and spatial selective attention. In R. Parasuraman (Ed.), The attentive brain (pp. 71-94). Cambridge, MA: MIT Press.

Awh, E., Jonides, J., Smith, E. E., Buxton, R. B., Frank, L. R., Love, T., . . . Gmeindel, L. (1999). Rehearsal in spatial working memory: Evidence from neuroimaging. Psychological Science, 10, 433 437. doi:10.1111/1467-9280.00182

Awh, E., Jonides, J., \& Reuter-Lorenz, P. A. (1998). Rehearsal in spatial working memory. Journal of Experimental Psychology: Human Perception and Performance, 24, 780-790. doi:10.1037/00961523.24.3.780

Bartels, A., \& Zeki, S. (2000). The architecture of the colour centre in the human visual brain: New results and a review. European Journal of Neuroscience, 12, 1172-1193.

Camperi, M., \& Wang, X.-J. (1998). A model of visuospatial working memory in prefrontal cortex: Recurrent network and cellular bistability. Journal of Computational Neuroscience, 5, 383405.

Churchland, P. S., \& Sejnowski, T. J. (1992). The computational brain. Cambridge, MA: MIT Press, Bradford Books.

Claeys, K. G., Dupont, P., Cornette, L., Sunaert, S., Van Hecke, P., De Schutter, E., \& Orban, G. A. (2004). Color discrimination involves ventral and dorsal stream areas. Cerebral Cortex, 14, $803-822$

Compte, A., Brunel, N., Goldman-Rakic, P. S., \& Wang, X.-J. (2000). Synaptic mechanisms and network dynamics underlying spatial working memory in a cortical network model. Cerebral Cortex, 10, 910-923.
Deco, G., \& Rolls, E. T. (2004). A neurodynamical model of visual attention and invariant object recognition. Vision Research, 44, 621-642.

Desimone, R., \& Duncan, J. (1995). Neural mechanisms of selective visual attention. Annual Review of Neuroscience, 18, 193-222. doi:10.1146/annurev.ne.18.030195.001205

Driver, J., \& Baylis, G. C. (1989). Movement and visual attention: The spotlight metaphor breaks down. Journal of Experimental Psychology: Human Perception and Performance, 15, 448-456. doi:10.1037/0096-1523.15.3.448

Duncan, J., \& Humphreys, G. W. (1989). Visual search and stimulus similarity. Psychological Review, 96, 433-458. doi:10.1037/0033295X.96.3.433

Duncan, J., Humphreys, G. W., \& Ward, R. (1997). Competitive brain activity in visual attention. Current Opinion in Neurobiology, 7, 255-261.

Edin, F., Klingberg, T., Johansson, P., McNab, F., Tegnér, J., \& Compte, A. (2009). Mechanism for top-down control of working memory capacity. Proceedings of the National Academy of Sciences, 106, 6802-6807. doi:10.1073/pnas.0901894106

Engebretson, P. H., \& Huttenlocher, J. (1996). Bias in spatial location due to categorization: Comment on Tversky and Schiano. Journal of Experimental Psychology: General, 125, 96-108. doi:10.1037/ 0096-3445.125.1.96

Fuster, J. M. (2003). Cortex and mind: Unifying cognition. Oxford, UK: Oxford University Press.

Goodale, M. A., \& Milner, A. D. (1992). Separate visual pathways for perception and action. Trends in Neurosciences, 15, 20-25. doi:10. 1016/0166-2236(92)90344-8

Hamker, F. H. (2005). The reentry hypothesis: The putative interaction of the frontal eye field, ventrolateral prefrontal cortex, and areas V4 and IT for attention and eye movements. Cerebral Cortex, 15, 431447.

Hillyard, S. A., \& Anllo-Vento, L. (1998). Event-related brain potentials in the study of visual selective attention. Proceedings of the National Academy of Sciences, 95, 781-787.

Hillyard, S. A., Vogel, E. K., \& Luck, S. J. (1998). Sensory gain control (amplification) as a mechanism of selective attention: Electrophysiological and neuroimaging evidence. Philosophical Transactions of the Royal Society B, 353, 1257-1270.

Hollingworth, A. (2009). Memory for real-world scenes. In J. R. Brockmole (Ed.), The visual world in memory (pp. 89-116). Hove, UK: Psychology Press.

Huang, L., \& Pashler, H. (2007). A Boolean map theory of visual attention. Psychological Review, 114, 599-631. doi:10.1037/0033-295X. 114.3.599

Hund, A. M., \& Spencer, J. P. (2003). Developmental changes in the relative weighting of geometric and experience-dependent location cues. Journal of Cognition and Development, 4, 338.

Huttenlocher, J., Hedges, L. V., \& Duncan, S. (1991). Categories and particulars: Prototype effects in estimating spatial location. Psychological Review, 98, 352-376. doi:10.1037/0033-295X.98.3. 352

Hyun, J.-S., \& Luck, S. J. (2007). Visual working memory as the substrate for mental rotation. Psychonomic Bulletin \& Review, 13, 154 158.

Itti, L., \& Koch, C. (2001). Computational modelling of visual attention. Nature Reviews Neuroscience, 2, 194-203. doi:10. $1038 / 35058500$

Just, M. A., \& Carpenter, P. A. (1992). A capacity theory of comprehension: Individual differences in working memory. Psychological Review, 98, 122-149. doi:10.1037/0033-295X. 99.1.122 
Kopecz, K., \& Schöner, G. (1995). Saccadic motor planning by integrating visual information and pre-information on neural, dynamic fields. Biological Cybernetics, 73, 49-60.

LaBerge, D., \& Brown, V. (1989). Theory of attentional operations in shape identification. Psychological Review, 96, 101-124. doi:10. 1037/0033-295X.96.1.101

LaBerge, D., Carlson, R. L., Williams, J. K., \& Bunney, B. G. (1997). Shifting attention in visual space: Tests of moving-spotlight models versus an activity-distribution model. Journal of Experimental Psychology: Human Perception and Performance, 23, 1380-1392. doi:10.1037/0096-1523.23.5.1380

Lipinski, J., Simmering, V. R., Johnson, J. S., \& Spencer, J. P. (2010a). The role of experience in location estimation: Target distributions shift location memory biases. Cognition, 115, 147-153.

Lipinski, J., Spencer, J. P., \& Samuelson, L. K. (2010b). Biased feedback in spatial recall yields a violation of delta rule learning. Psychonomic Bulletin \& Review, 17, 581-588. doi:10.3758/PBR.17.4.581

Liu, T., \& Mance, I. (2011). Constant spread of feature-based attention across the visual field. Vision Research, 51, 26-33. doi:10.1016/j. visres.2010.09.023

Liu, T., Slotnick, S. D., Serences, J. T., \& Yantis, S. (2003). Cortical mechanisms of feature-based attentional control. Cerebral Cortex, 13, 1334-1343

Liverence, B. M., \& Scholl, B. J. (2011). Selective attention warps spatial representation: Parallel but opposing effects on attended versus inhibited objects. Psychological Science, 22, 1600-1608.

Luck, S. J., \& Vecera, S. P. (2002). Attention. In H. P. (Series Ed.) \& S. Y. (Volume Ed.) (Eds.), Steven's Handbook of experimental psychology: Vol. 1. Sensation and perception (3rd ed., pp. 235-286). New York, NY: Wiley.

Maunsell, J. H. R., \& Treue, S. (2006). Feature-based attention in visual cortex. Trends in Neurosciences, 29, 317-322. doi:10.1016/j.tins. 2006.04.001

Mozer, M. C., \& Sitton, M. (1998). Computational modeling of spatial attention. In H. Pashler (Ed.), Attention (pp. 341-393). London, UK: UCL Press.

Neisser, U. (1967). Cognitive psychology. New York, NY: AppletonCentury-Crofts.

Posner, M. I. (1980). Orienting of attention. Quarterly Journal of Experimental Psychology, 32, 3-25. doi:10.1080/ 00335558008248231

Postle, B. R., Awh, E., Jonides, J., Smith, E. E., \& D'Esposito, M. (1999). The in which and how of attention-based rehearsal in spatial working memory. Cognitive Brain Research, 20, 194-205.

Riggall, A. C., \& Postle, B. R. (2012). The relationship between working memory storage and elevated activity as measured with functional magnetic resonance imaging. Journal of Neuroscience, 32, 12990 12998.

Roggeman, C., Klingberg, T., Feenstra, H. E. M., Compte, A., \& Almeida, R. (2014). Trade-off between capacity and precision in visuospatial working memory. Journal of Cognitive Neuroscience, 26, 211-222. doi:10.1162/jocn_a_00485

Schiano, D. J., \& Tversky, B. (1992). Structure and strategy in encoding simplified graphs. Memory \& Cognition, 20, 12-20.

Schneegans, S. (2016). Sensory-motor and cognitive transformations. In G. Schöner, J. P. Spencer, \& the DFT Research Group (Eds.), Dynamic thinking: A primer on dynamic field theory (pp. 169196). New York, NY: Oxford University Press.

Schneegans, S., Spencer, J. P., Schöner, G., Hwang, S., \& Hollingworth, A. (2014). Dynamic interactions between visual working memory and saccade target selection. Journal of Vision, 14(11), 9:1-23. doi: $10.1167 / 14.11 .9$

Schutte, A. R., \& Spencer, J. P. (2009). Tests of the dynamic field theory and the spatial precision hypothesis: Capturing a qualitative developmental transition in spatial working memory. Journal of
Experimental Psychology: Human Perception and Performance, $35,1698-1725$.

Schutte, A. R., Spencer, J. P., \& Schöner, G. (2003). Testing the dynamic field theory: Working memory for locations becomes more spatially precise over development. Child Development, $74,1393-1417$.

Serences, J. T., \& Boynton, G. M. (2007). Feature-based attentional modulations in the absence of direct visual stimulation. Neuron, 55, 301312.

Sheth, B. R., \& Shimojo, S. (2001). Compression of space in visual memory. Vision Research, 41, 329-341. doi:10.1016/S00426989(00)00230-3

Spencer, J. P., \& Hund, A. M. (2002). Prototypes and particulars: Spatial categories are formed using geometric and experience-dependent information. Journal of Experimental Psychology: General, 131, $16-37$.

Spencer, J. P., Perone, S., \& Johnson, J. S. (2009). The dynamic field theory and embodied cognitive dynamics. In J. P. Spencer, M. S. Thomas, \& J. L. McClelland (Eds.), Toward a new grand theory of development? Connectionism and dynamic systems theory re-considered. New York, NY: Oxford University Press.

Spencer, J. P., Simmering, V. R., Schutte, A. R., \& Schöner, G. (2007) What does theoretical neuroscience have to offer the study of behavioral development? Insights from a dynamic field theory of spatial cognition. In J. M. Plumert \& J. P. Spencer (Eds.), The emerging spatial mind (pp. 320-361). New York, NY: Oxford University Press.

Spencer, J. P., Simmering, V. R., \& Schutte, A. R. (2006). Toward a formal theory of flexible spatial behavior: Geometric category biases generalize across pointing and verbal response types. Journal of Experimental Psychology: Human Perception and Performance, 32, 473-490. doi:10.1037/0096-1523.32.2.473

Treisman, A. M., \& Gelade, G. (1980). A feature-integration theory of attention. Cognitive Psychology, 12, 97-136. doi:10.1016/00100285(80)90005-5

Tversky, B., \& Schiano, D. J. (1989). Perceptual and conceptual factors in distortions in memory for graphs and maps. Journal of Experimental Psychology: General, 118, 387-398. doi:10.1037/0096-3445.118.4. 387

Ungerleider, L. G., \& Mishkin, M. (1982). Two cortical visual systems. In D. J. Ingle, M. A. Goodale, \& R. J. W. Mansfield (Eds.), Analysis of visual behavior (pp. 549-586). Cambridge, MA: MIT Press.

Van der Stigchel, S., Merten, H., Meeter, M., \& Theeuwes, J. (2007). The effects of a task-irrelevant visual event on spatial working memory. Psychonomic Bulletin \& Review, 14, 1066-1071. doi:10.3758/ BF03193092

Wang, X.-J. (2001). Synaptic reverberation underlying mnemonic persistent activity. Trends in Neurosciences, 24, 455-463.

Wei, Z., Wang, X.-J., \& Wang, D.-H. (2012). From distributed resources to limited slots in multiple-item working memory: A spiking network model with normalization. Journal of Neuroscience, 32, 11228-11240. doi:10.1523/JNEUROSCI. 0735-12.2012

Werner, S., \& Schmidt, T. (2000). Investigating spatial reference systems through memory distortions. In C. Freska, W. Brauer, C. Habel, \& K. F. Wender (Eds.), Spatial cognition II: Integrating abstract theories, emprirical studies, formal methods, and practical applications (pp. 169-183). Berlin, Germany: Springer.

Williams, R. J. (1986). The logic of activation functions. In D. E. Rumelhart, J. L. McClelland, \& the P. R. Group (Eds.), Parallel distributed processing: Volume 1 (pp. 423-443). Cambridge, MA: MIT Press. 
Wolfe, J. M. (1994). Guided Search 2.0: A revised model of visual search. Psychonomic Bulletin \& Review, 1, 202-238. doi:10.3758/BF03200774

Xiao, Y. (2014). Hierarchy of hue maps in the primate visual cortex. Journal of Ophthalmic \& Vision Research, 9, 144-147.

Xiao, Y., Wang, Y., \& Felleman, D. J. (2003). A spatially organized representation of colour in macaque cortical area V2. Nature, 421, $532-539$.
Zhong, Y.-M., \& Rockland, K. S. (2003). Inferior parietal lobule projections to anterior inferotemporal cortex (area TE) in macaque monkey. Cerebral Cortex, 13, 527-540.

Zibner, S. K. U., \& Faubel, C. (2016). Dynamic scene representations and autonomous robotics. In G. Schöner, J. P. Spencer, \& the DFT Research Group (Eds.), Dynamic thinking: A primer on dynamic field theory (pp. 227-246). New York, NY: Oxford University Press. 